ARTICLE

Received 23 Nov 2015 | Accepted 3 May 2016 | Published 10 Jun 2016

DOI: $10.1038 /$ ncomms11808

OPEN

\title{
Cholesterol and ORP1L-mediated ER contact sites control autophagosome transport and fusion with the endocytic pathway
}

\author{
Ruud H. Wijdeven ${ }^{1}$, Hans Janssen ${ }^{1}$, Leila Nahidiazar ${ }^{1}$, Lennert Janssen ${ }^{1,2}$, Kees Jalink ${ }^{1}$, Ilana Berlin ${ }^{1,2}$ \\ $\&$ Jacques Neefjes ${ }^{1,2}$
}

\begin{abstract}
Autophagy is the main homeostatic pathway guiding cytosolic materials for degradation by the lysosome. Maturation of autophagosomes requires their transport towards the perinuclear region of the cell, with key factors underlying both processes still poorly understood. Here we show that transport and positioning of late autophagosomes depends on cholesterol by way of the cholesterol-sensing Rab7 effector ORP1L. ORP1L localizes to late autophagosomes and-under low-cholesterol conditions-contacts the ER protein VAP-A, forming ER-autophagosome contact sites, which prevent minus-end transport by the Rab7-RILP-dynein complex. ORP1L-mediated contact sites also inhibit localization of PLEKHM1 to Rab7. PLEKHM1, together with RILP, then recruits the homotypic fusion and vacuole protein-sorting (HOPS) complex for fusion of autophagosomes with late endosomes and lysosomes. Thus, ORP1L, via its liganding by lipids and the formation of contacts between autophagic vacuoles and the ER, governs the last steps in autophagy that lead to the lysosomal degradation of cytosolic material.
\end{abstract}

\footnotetext{
${ }^{1}$ Division of Cell Biology, The Netherlands Cancer Institute, Plesmanlaan 121, 1066 CX Amsterdam, The Netherlands. ${ }^{2}$ Department of Chemical Immunology, Leiden University Medical Center, 2300 RC Leiden, The Netherlands. Correspondence and requests for materials should be addressed to J.N. (email: j.neefjes@nki.nl).
} 
A utophagy is the primary pathway for degradation of cytosolic material, protein aggregates and damaged organelles in lysosomes (Lys), with its de-regulation implicated in neurodegenerative diseases, compromised immunity and cancer $^{1-3}$. Cargo destined for such proteolysis becomes sequestered into double-membraned autophagosomes, lined with the distinguishing modifier LC3. Following membrane-closure these structures fuse with lysosomes, giving rise to a hybrid organellethe autolysosome. Alternatively, autophagosomes progress through an intermediate amphisome stage-the result of fusion with late endosomes (LEs)—and subsequently mature by acquiring lysosomal characteristics or merge with the pre-existing lysosomes in a secondary fusion event ${ }^{4}$. While many of the essential molecular players for these processes have been identified, our understanding of the underlying factors controlling transport and fusion of autophagic vacuoles (AVs, which include autophagosomes, amphisomes and autolysosomes) remains incomplete.

Entry of autophagic cargoes into the endocytic system is orchestrated by components from both the autophagosome and the late endosomal/lysosomal (LE/Ly) side. A complex consisting of autophagosome-localized ATG14L, the SNAREs STX17 and SNAP29 as well as endosomal VAMP8 has recently been shown to execute the heterologous autophagosome/LE (or autophagosome/Ly) fusion event ${ }^{5-8}$. Tethering of the participating membranes, which precedes fusion, critically depends on both STX17 (ref. 6) and the GTPase Rab7 (refs 9-12), with the latter likely provided by the endosome. STX17 has been shown to recruit the homotypic fusion and vacuole protein-sorting (HOPS) complex, which is thought to bridge the two organelles, thus facilitating their unification by SNARE proteins ${ }^{13-16}$. In addition, PLEKHM1 as well as RILP-both effectors of Rab7-can bind HOPS ${ }^{15,17-20}$, with PLEKHM1 reportedly involved in autophagosome-LE/Ly fusion ${ }^{17}$. The above tethering and fusion proteins are recruited to the autophagosome/LE (or Ly) interface in a stimulus-dependent manner ${ }^{14,17}$, suggesting existence of additional factors to influence and fine-tune the fusion process.

Entry of autophagosomes into the late endosomal/lysosomal pathway requires dynein motor-mediated transport towards the microtubule organizing center ${ }^{21-26}$, where most lysosomes reside. However, the nature of motor recruitment control and its effects on transport of autophagosomes is largely unknown. During maturation, autophagosomes acquire Rab7, typically relegated to the LE/Ly $9,10,27$, perhaps as a result of fusion with these compartments. On endosomes, Rab7 can associate with several effectors, including RILP, which recruits the dynein-dynactin motor for minus-end transport ${ }^{28,29}$. Whether autophagosomes utilize this Rab7-associated transport and fusion machinery en route to the lysosome, and if so, what factors control this process, is not known.

Here we show that transport of autophagosomes is governed by two Rab7 effectors-RILP and the cholesterol-sensor ORP1L. ORP1L localizes to amphisomes and autolysosomes and, under low cholesterol conditions, contacts the ER protein VAP-A to form intimate membrane contact sites (MCSs) with the ER. These ER-autophagosome MCSs prevent dynactin binding to RILP, thus blocking dynein-mediated transport. Furthermore, ORP1L in its low-cholesterol state acts as a negative regulator of AV/LE fusion and prevents binding of both PLEKHM1 and HOPS by Rab7-RILP. Thus, our study reveals a molecular mechanism whereby ORP1L, via its liganding by lipids and the formation of contacts between AVs and the ER, controls the degradation of cytosolic components by autophagy.

\section{Results}

Cholesterol controls autophagosome positioning. Autophagic vacuoles are dynamic compartments that move bi-directionally along microtubules $20,21,28$ and mostly encounter lysosomes in the perinuclear region ${ }^{30}$. Intriguingly, steady-state cellular distribution of AVs varies greatly between different cell types. While in cervical HeLa cells AVs are dispersed throughout the cytoplasm, in melanoma MelJuSo cells most structures containing the autophagy marker LC3 localize at the perinuclear region (Fig. 1a). Since intracellular AV position is determined by the activities of kinesin and dynein motor proteins ${ }^{21,22,31}$ common to all cells, these differences might arise from variations in motor activity or recruitment. Strikingly, MelJuSo cells also contain more (endosomal) cholesterol (Fig. 1a), known to promote localization of endosomes and lysosomes at the microtubule minus-end ${ }^{32,33}$, close to the nucleus, raising the possibility that AV transport is modulated by endosomal cholesterol levels.

To test this, we reduced cholesterol levels in cells using statin (to inhibit endogenous cholesterol production, supplemented with mevalonate to provide essential nonsteroidal isoprenoids) and lipid-free serum (to inhibit the major source of cholesterol for cells as taken up through the LDL receptor) ${ }^{34}$. In addition, we accumulated cholesterol in the endosomal compartment by exposure of cells to U18666A (refs 32,34), an inhibitor of the endosomal cholesterol exporter NPC1 (ref. 35). Strikingly, in the case of lipid depletion, AVs were found to scatter throughout the cytoplasmic space and accumulate at the tips of the cell, while they concentrated at the perinuclear region under conditions resulting in accumulated endosomal cholesterol. This was observed both in MelJuSo and HeLa cells, as well as for statin and lipid-free serum separately, ruling out non-cholesterolmediated effects caused by statin (Fig. 1b; Supplementary Fig. 1a,b). To follow the dynamics of these AVs, we performed live-cell imaging using mCherry-LC3 and Lysotracker to label AVs and the late endocytic compartments. This indicated that the AVs localizing to the tips under low-cholesterol conditions were relatively static and remained located at their position for prolonged periods of time (Fig. 1c; Supplementary Movies 1-3), suggesting a defect in microtubule-based minus-end transport. Furthermore, the majority of these peripheral AVs labelled positive for Lysotracker (Fig. 1d and Supplementary Movies 1-3), as well as for Rab7 (Supplementary Fig. 1c), defining them as late or mature autophagosomes (amphisomes and autolysosomes). Thus, manipulating cholesterol affects the transport dynamics of late autophagosomes.

ORP1L senses cholesterol to regulate autophagosome transport. A key distinguishing characteristic between early and late AVs is the presence of Rab7 (ref. 36), suggesting that Rab7 effectors could be responsible for cholesterol-dependent late autophagosome re-localization. We, and others, have previously shown that the Rab7 effector ORP1L acts as a cholesterol sensor on late endosomes ${ }^{33,37}$. Upon (membrane) cholesterol binding by its ORD domain, ORP1L maintains a conformation compatible with dynein motor recruitment and minus-end transport, while absence of cholesterol prevents this ${ }^{33,37}$. Silencing ORP1L in MelJuSo cells indeed reduced the fraction of AVs localizing at the cell periphery under basal conditions (Fig. 2a; Supplementary Fig. 2a). Furthermore, ORP1L knockdown strongly inhibited AV scattering caused by cholesterol depletion (Fig. 2a), illustrating a critical role for ORP1L in cholesterol-mediated AV repositioning. To test whether cholesterol controls positioning directly via ORP1L, we expressed GFP-tagged wild-type ORP1L or variants altering its cholesterol-sensing properties (Fig. 2b). These were: $\triangle \mathrm{ORD}$, lacking the cholesterol-interacting ORD domain (thereby mimicking the non-cholesterol bound state of ORP1L and acting as a cholesterol-independent inhibitor of dynein recruitment, since this variant cannot be inactivated by cholesterol) and 
a
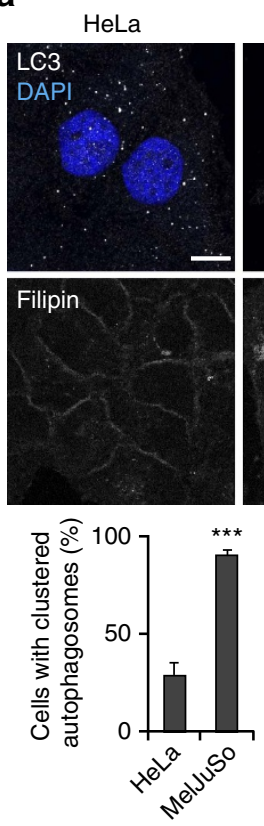

C
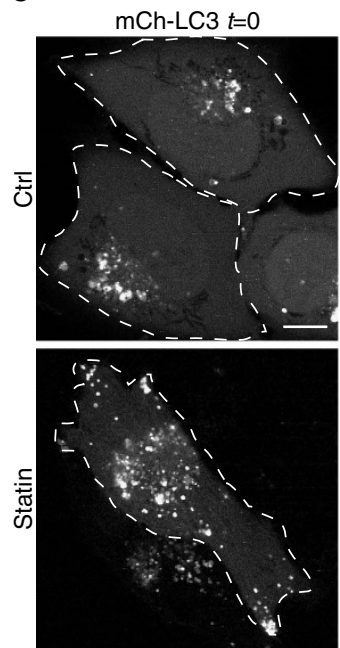

MelJuSo
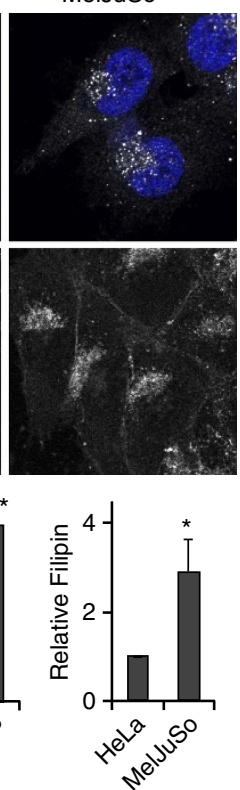

b
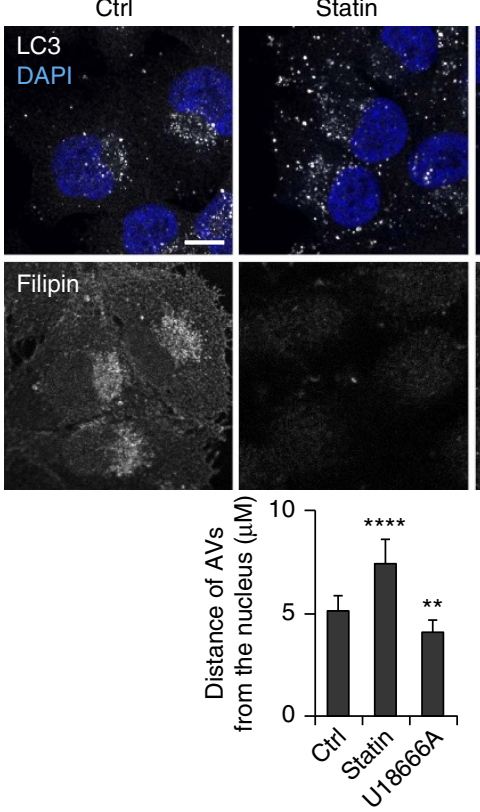

d
U18666A
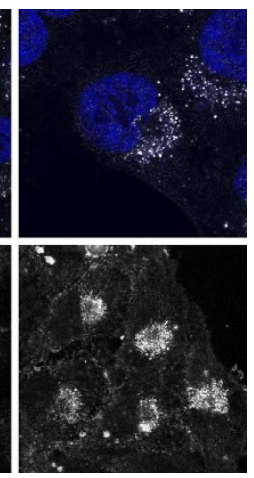

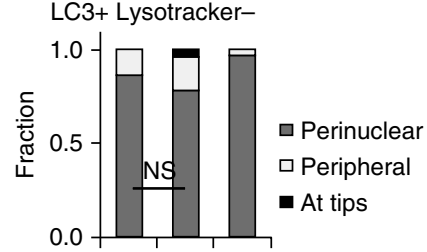

LC3+ Lysotracker+

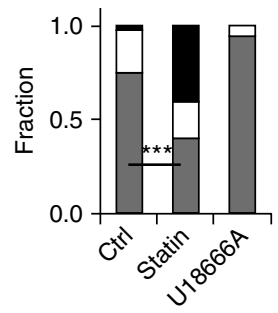

Figure 1 | Cholesterol modulates autophagosome positioning. (a) HeLa or MelJuSo cells were stained for LC3 and 4,6-diamidino-2-phenylindole (DAPI), or with filipin to detect cholesterol. Scale bar, $10 \mu \mathrm{m}$. Panels below: quantification for the presence of a detectable perinuclear LC3 cluster: $>100$ cells were analysed per experiment. For filipin labelling, signal intensity was measured for at least three fields per experiment and was normalized to signal intensity in HeLa cells, which was set at 1. Bars indicate mean + s.d. from independent triplicates, significance calculated using Student's t-test. (b) MelJuSo cells cultured for $24 \mathrm{~h}$ under cholesterol-depleting conditions (see Methods) or exposed for $24 \mathrm{~h}$ to $3 \mu \mathrm{M}$ U18666 were stained for LC3 and DAPI. Scale bar, $10 \mu \mathrm{m}$. Lower panel: quantification of the average distance $(\mu \mathrm{m})$ of the AVs from the nucleus (using the Las AF software), from $>10$ cells per experiment. Bars indicate mean + s.d. from independent triplicates, significance calculated using Student's $t$-test. (c) Distribution and dynamics of mCherry-LC3marked vesicles in control versus lipid-depleted MelJuSo cells. Confocal image at start of time lapse is shown on the left, vesicle trajectories over a $480-\mathrm{s}$ interval are displayed on the right. Colours of individual vesicle trajectories reflect maximal displacement rate (blue $=0$, red $=0.8 \mu \mathrm{ms}{ }^{-1}$ ) achieved during the 480-s interval. Scale bar, $10 \mu \mathrm{m}$. (d) Quantification of the localization of mCherry-LC3 vesicles either or not labelling for Lysotracker in MelJuSo cells treated as indicated. At least five cells per replicate were quantified, bars indicate mean + s.d. from independent triplicates. Significance calculated using Student's $t$-test (Supplementary Movies 1-3). NS, not significant, ${ }^{\star} P<0.05,{ }^{\star \star} P<0.01,{ }^{\star \star \star} P<0.001,{ }^{\star \star \star \star} P<0.0001$ ). AV, autophagic vacuole.

$\triangle$ ORDPHDPHD, where the ORD domain is replaced by a tandem membrane-targeting $\mathrm{PH}$ domain (PHD) to reflect the membrane-bound high cholesterol state (acting as a cholesterol-independent permissive factor for dynein binding, since the tandem PHD domains remain membrane bound and allow dynein recruitment, mimicking the high cholesterol state even under conditions of low cholesterol) $)^{33}$ (modelled in Supplementary Fig. 2b). These constructs mimic the low or high late endosomal cholesterol states, respectively, independently of the actual cholesterol concentration, since the ORD domain has been removed ${ }^{33}$. Expression of ORP1L and especially ORP1L- $\triangle$ ORD, but not ORP1L- $\triangle$ ORDPHDPHD, resulted in profound scattering of AVs (Fig. 2c,d), indicating that ORP1L regulates $\mathrm{AV}$ positioning as a function of its cholesterol-bound state. Importantly, cholesterol depletion induced scattering of AVs in cells ectopically expressing GFP, but had no effect on cells expressing ORP1L- $\triangle$ ORDPHDPHD, implying that low cholesterol levels had the effect through the ORP1L protein 
a
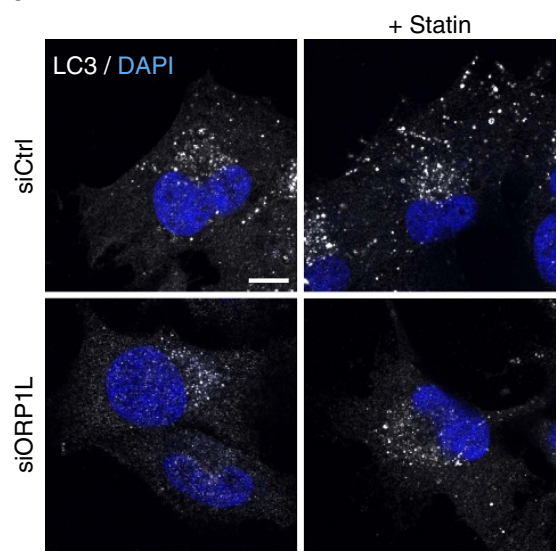

d
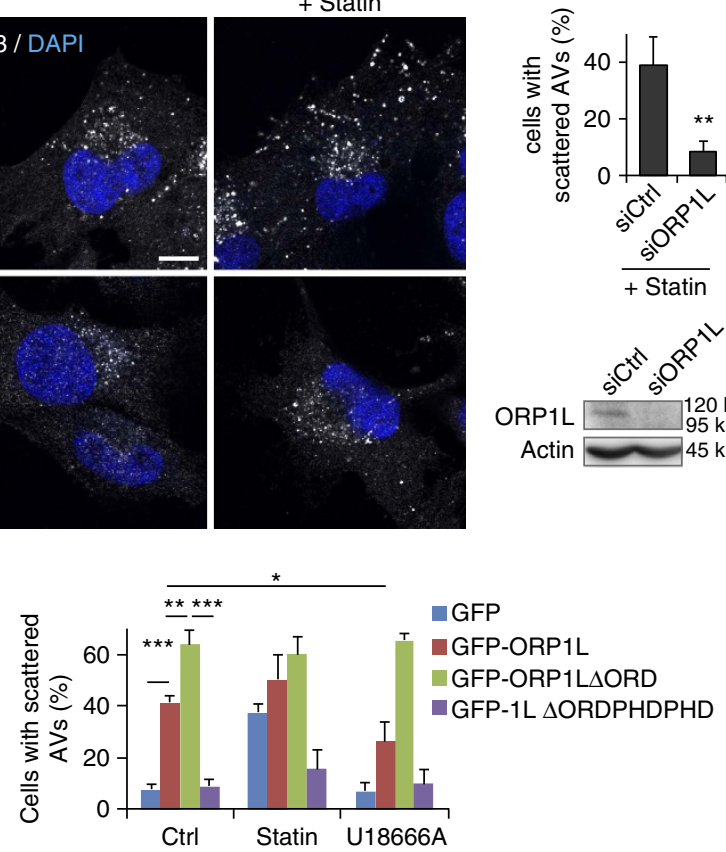

Actin b

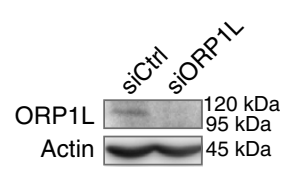

e
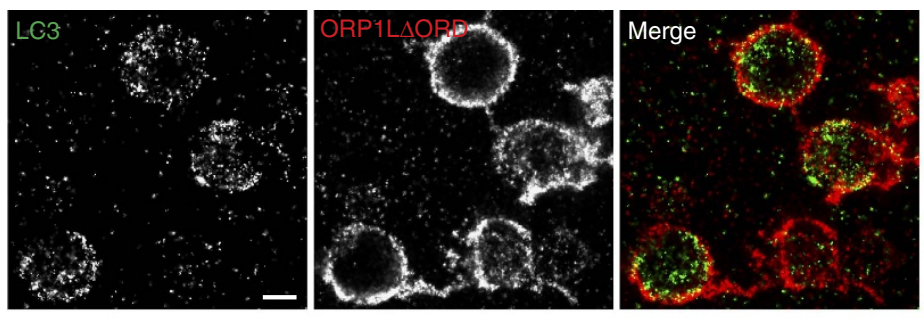

g
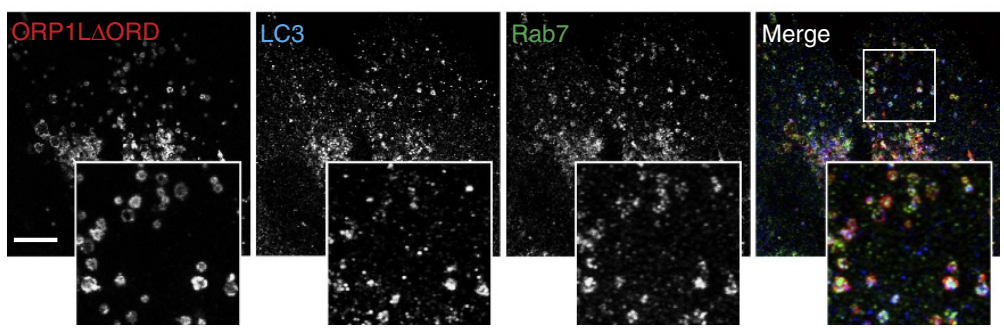

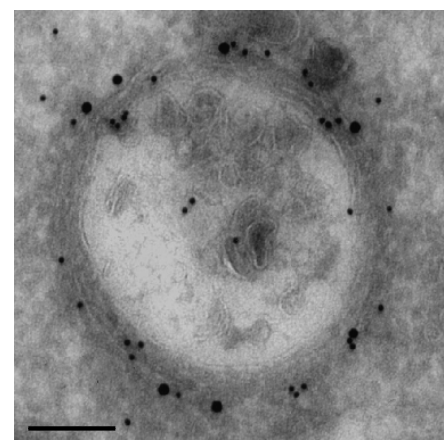

- HA-LC3

- GFP-ORP1L $\triangle \mathrm{ORD}$

Figure 2 | ORP1L senses cholesterol to control autophagosome positioning. (a) MelJuSo cells transfected with siCtrl or siORP1L were either or not cultured under lipid-depleted conditions and stained for LC3 and DAPI. Scale bar, $10 \mu \mathrm{m}$. Top right: quantification of scattering of LC3 structures under lipiddepleted conditions, over $>100$ cells were counted per experiment, bars indicate mean + s.d. from three experiments. Means were compared using Student's $t$-test. Lower right: western blot to validate silencing of ORP1L. (b) Overview of the ORP1L constructs and their domains used in this study. The C-terminal ORD domain binds oxysterols. (c) MelJuSo cells transfected with the indicated GFP-tagged constructs were fixed and stained for LC3 and DAPI. Scale bar, $10 \mu \mathrm{m}$. (d) Quantification of cells with scattered autophagic vesicles in cells under different cholesterol manipulation conditions and expressing different GFP-tagged constructs, as shown in $\mathbf{d}$ and Supplementary Fig. 2b. Quantification was performed on $>50$ transfected cells per experiment, bars indicate mean + s.d. from independent triplicates. Significance calculated using Student's $t$-test. (e) Superresolution microscopy on cells expressing HAORP1LAORD, stained for HA and LC3, as indicated. Scale bar, $500 \mathrm{~nm}$ (f) Cryo-immuno-EM on HeLa cells expressing HA-LC3 and GFP-ORP1L $\triangle$ ORD stained with $\mathrm{HA}^{10 \mathrm{~nm}}$ and GFP15 $\mathrm{nm}$ gold antibodies, respectively. Scale bar, $100 \mathrm{~nm}$. (g) MelJuSo cells expressing GFP-ORP1L $\triangle O R D$ were fixed and stained for Rab7 and LC3. Scale bar, $10 \mu \mathrm{m} .{ }^{\star} P<0.05,{ }^{\star \star} P<0.01,{ }^{\star \star \star} P<0.001$. AV, autophagic vacuole.

(Fig. 2d and Supplementary Fig. 2c). In line with this, GFPORP1L and even more pronounced the ORP1LAORD construct resisted AV clustering following cholesterol accumulation by U18666A, unlike GFP or the ORP1LAORDPHDPHD construct mimicking the high-cholesterol form of ORP1L (Fig. 2d; Supplementary Fig. 2c). While cholesterol manipulation of cells may result in several effects on lipids and proteins, the fact that the low- and high-cholesterol state configurations of ORP1L override these effects suggests that the effects of cholesterol on AV positioning are largely ORP1L mediated.
To investigate which AV substructures are subject to regulation by ORP1L, we investigated the types of structures ORP1L localized to. As assessed by super-resolution total internal reflection (TIRF) microscopy, ORP1L localized to the limiting membrane of AVs, (Fig. 2e), which was confirmed by cryo-immuno-EM, where ORP1L was found to localize to single-membrane AVs, which defines them as amphisomes or autolysosomes (Fig. 2f). In support of this, all ORP1L marked AVs co-labelled for Rab7 (Fig. 2g; Supplementary Fig. 2d). Together, these data suggest that cholesterol as sensed by ORP1L 
dictates the intracellular position of Rab7-marked late autophagosomes.

Low cholesterol and ORP1L yield of ER-AV contact sites. When recruited to LE by Rab7, ORP1L has a conformational state allowing interactions with ER-protein VAP-A to establish a MCS between these two compartments. VAP-A then controls the binding of the dynein motor to RILP ${ }^{33,38}$. This interaction with VAP-A is stabilized by mutating or deleting the ORD domain of ORP1L and is promoted by low cholesterol culture conditions $^{38}$ (Fig. 3a). To assess whether ORP1L has the ability to form physical contacts between late autophagosomes and the ER, coincidence of ER marker calnexin and LC3 was detected as a function of ORP1L mutants. Robust distribution of (calnexin positive) ER pockets to $\mathrm{LC}^{+}{ }^{+}$structures occurred in the presence of either ORP1L or ORP1L $\triangle$ ORD, and could be prevented by eliminating the VAP-A-binding site FFAT (two phenylalanines (FF) in an Acidic Tract) in the latter (by mutating Y477 and D478 into alanines, Fig. 3a). Typical MCSs are characterized by a spatial separation of less than $10-30 \mathrm{~nm}$ between two compartments ${ }^{39}$, which required analyses of these contact sites by EM and superresolution light microscopy. Cryo-immuno-EM showed long stretches of ER closely positioned to vesicles positive for both ORP1L and LC3 (Fig. 3b; Supplementary Fig. 3a). 3-Colour superresolution TIRF microscopy confirmed the existence of VAP-A-ORP1L contacts on LC3 positive structures, with AVs containing ORP1L significantly surrounded by ER protein VAPA (Fig. 3c). At this level of resolution, ORP1L was again found at the limiting membrane and surrounded by VAP-A, covering major parts of the vesicle (Supplementary Movie 4).

Similar to overexpression of ORP1L and ORP1L $\Delta$ ORD, cholesterol depletion profoundly stimulated re-distribution of the ER towards LC3 puncta, creating a distinct punctate ER contacting the $\mathrm{LC}^{+}{ }^{+}$structures (Fig. 3d; Supplementary Fig. 3b). Notably, silencing of either ORP1L or VAP-A reduced these ER contacts with LC3 in the cell periphery, while perinuclear MCSs were only sensitive to silencing VAP-A (Supplementary Fig. 3c). This suggests that cholesterol controls the formation of ORP1L-VAP-A contact sites, as well as contact sites between VAP-A and a different autophagosomal protein. Together, these results visualized a novel membrane contact site, one between late autophagosomes and the ER.

RILP regulates transport of late autophagosomes. On LE/Ly, ORP1L forms a tripartite complex with Rab7 and RILP and controls binding of the dynein motor complex to RILP ${ }^{33,40}$. RILP is also found on AVs and its recruitment to Rab7 is enhanced under conditions that promote the autophagic flux ${ }^{41}$, suggesting that RILP could also drive dynein acquisition on late autophagosomes-and is by extension sensitive to ORP1L. To assess this, we silenced RILP in MelJuSo cells, which yielded AV scattering and accumulation in the tips of the cell (Fig. 4a,b). Conversely, ectopic expression of RILP in HeLa cells produced strong clustering of $\mathrm{AVs}$ in the perinuclear region (Fig. 4c,d), while a RILP mutant deficient in dynein-dynactin interactions ${ }^{40}$ ( $\Delta 199)$ left the AVs scattered in the cell periphery. Furthermore, RILP induced recruitment of the dynein-dynactin subunit p150glued to AVs (Supplementary Fig. 4a). RILP-mediated AV repositioning is dynein dependent, as disruption of the dynein motor complex by ectopically expressing p50 dynamitin (ref. 42), which by itself accumulated AVs at the tips of cells (Supplementary Fig. 4b), negated the perinuclear clustering induced by RILP (Fig. 4e). RILP localized to a fraction of the $\mathrm{LC}^{+}$vesicles (Fig. 4e), constituting the Rab7-marked late autophagosomal pool (Fig. 4f). This was further supported by
immuno-EM analysis that showed $\operatorname{RILP}(\Delta 199)$ (full-length RILP strongly clustered AVs, LEs and Lys in the perinuclear region, thus preventing proper analyses) localizing to the limiting membrane of single membrane autophagosomes (amphisomes and autolysosomes ${ }^{43}$; Fig. 4g). These data suggest that the Rab7 effector RILP recruits dynein for minus-end transport of mature autophagosomes.

To assess the effect of ORP1L on RILP-mediated transport, we co-expressed RILP with ORP1L. While overexpression of ORP1L had only minor effects on RILP-induced perinuclear clustering of AVs (Fig. 4h,i), ORP1L $\triangle$ ORD completely eliminated this clustering, an effect dependent on its ability to bind VAP-A, since mutating the interacting FFAT motif in ORP1L $\triangle$ ORD restored RILP-induced perinuclear clustering. Similar to this, recruitment of dynactin subunit p150 glued to AVs was blocked by co-expression of RILP with ORP1L $\triangle$ ORD (Supplementary Fig. 4a). These data indicate that Rab7-effector RILP recruits the dynein motor to late autophagosomes for minus-end transport, and that ORP1L further controls this transport via the formation of ER-contact sites with VAP-A.

ORP1L controls autophagosome maturation. Since transport of AVs is associated to their maturation, we reasoned that ORP1L might inhibit the autophagic flux. Consistent with this, ORP1L silencing reduced the number of $\mathrm{LC}^{+}$structures in cells (Figs 2a and 5a), as well as overall LC3B-II and p62 levels (Fig. 5b). This decrease was negated by blocking lysosomal degradation (Fig. 5b), illustrating that ORP1L depletion spurs the autophagic flux. Decreased p62 levels could imply that ORP1L also controls clearance of ubiquitinated protein aggregates. Aggregate-like induced structures are formed on cellular stress and recognized by selective autophagy receptors, including p62, for targeting to the autophagic pathway. While ORP1L knockdown had no effect on the generation of puromycin-induced aggregate-like induced structures, the number of cells containing ubiquitinated aggregates at 4 and $6 \mathrm{~h}$ following puromycin washout was reduced when compared with control cells (Fig. 5c). At these time points, most remaining aggregates stained positive for LC3, indicating effective targeting to the autophagosomal pathway. Thus, silencing of ORP1L increases the autophagic flux and the rate of protein aggregate degradation.

Conversely, ectopic expression of ORP1L increased the number of AVs in cells, which was more pronounced by expressing ORP1L $\triangle$ ORD (Fig. 5d). This phenotype was largely corrected for by mutating the VAP-A interacting FFAT motif in ORP1L $\triangle$ ORD. Co-staining with the LE/Ly marker CD63 revealed that overexpression of either ORP1L or ORP1L $\triangle$ ORD increased the numbers of both early $\left(\mathrm{LC}^{+}{ }^{+} \mathrm{CD} 63^{-}\right)$and late $\left(\mathrm{LC}^{+}{ }^{+} \mathrm{CD} 63^{+}\right)$ autophagosomes. Surprisingly, the most significant difference was observed for the early (ORP1L negative) autophagosomes, suggesting that in addition to fine-tuning dynein motor-driven transport, ORP1L controls autophagosome fusion with the LE/Ly. To test this, we used the tandem mRFP-GFP-LC3B construct that contains a $\mathrm{pH}$ insensitive $\mathrm{mRFP}$ and a $\mathrm{pH}$ sensitive GFP molecule to distinguish early $\left(\mathrm{GFP}^{+} \mathrm{RFP}^{+}\right)$from late $\left(\mathrm{GFP}^{-} \mathrm{RFP}^{+}\right)$more acidic autophagosomes ${ }^{12}$. Following amino-acid starvation to accelerate the autophagic flux, cells expressing the low cholesterol mimic ORP1L $\triangle$ ORD harboured a significantly elevated fraction of early autophagosomes when compared with cells expressing wild-type ORP1L and vector control (Fig. 5e). Thus, ORP1Linduced ER contact sites are able to modulate autophagosome maturation.

We then tested whether cholesterol depletion or accumulation would also affect the autophagic flux. Whereas cholesterollowering conditions did not yield measurable changes in 
a
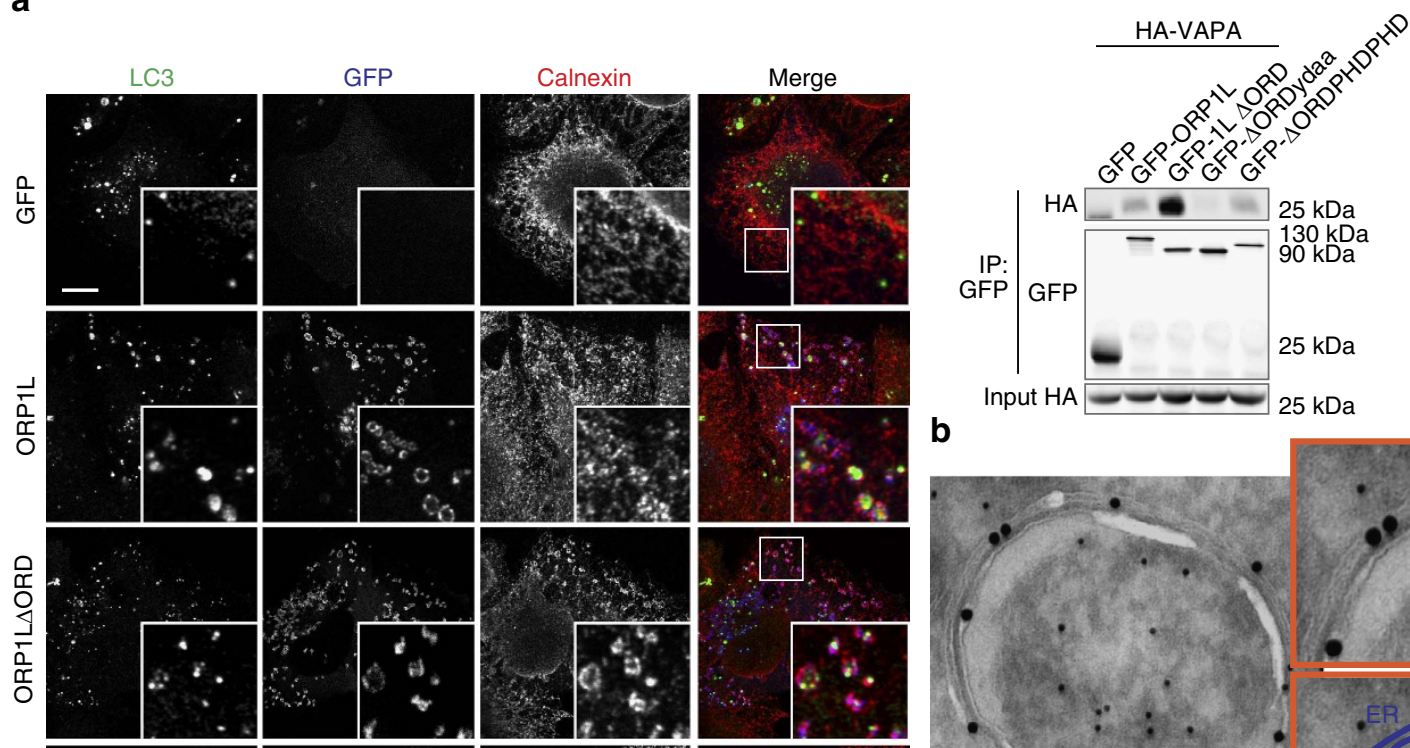

b
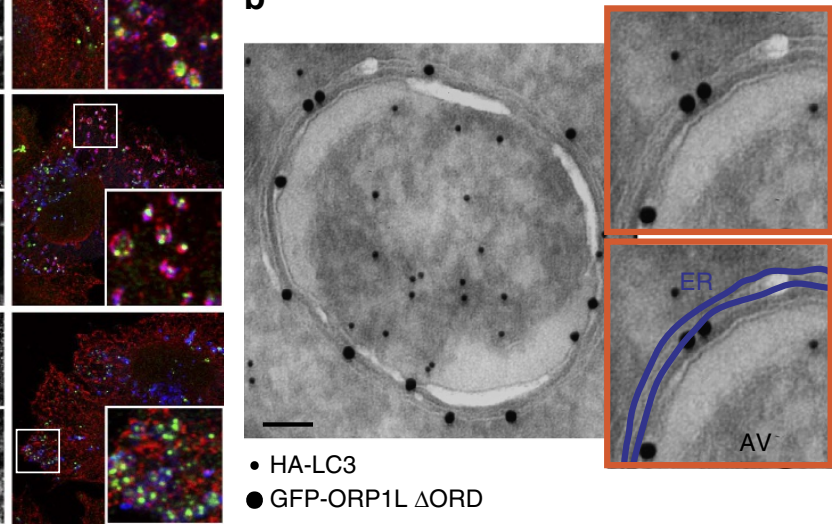

- GFP-ORP1L $\triangle$ ORD

d
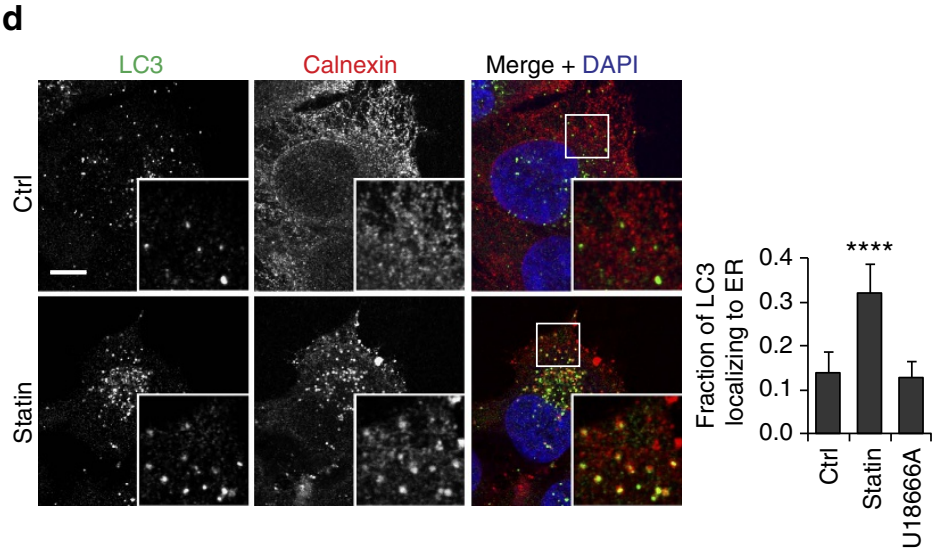

C
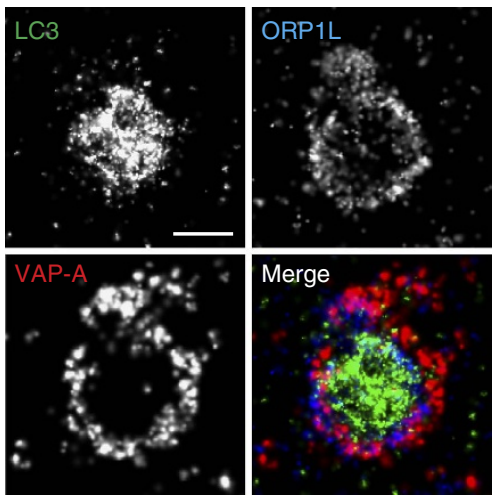

Figure 3 | ORP1L and VAP-A form cholesterol-dependent ER-AV contact sites (a) MelJuSo cells expressing GFP or GFP-tagged ORP1L mutants were fixed and stained for LC3 and Calnexin. Y477 and D478 in the FFAT motif were mutated to alanines (A) in the ORP1L ydaa mutant. Scale bar, $10 \mu \mathrm{m}$. Right: co-immunoprecipitation for ORP1L (mutants) with VAP-A. GFP-ORP1L mutants or GFP were isolated from lysates of HEK293T cells co-overexpressing HAVAPA using GFP-Trap beads. Western blot filters were probed for isolated GFP-tagged proteins, the associated HA-VAP-A and the input HA-VAP-A, as indicated. (b) Cryo-immuno-EM on HeLa cells expressing HA-LC3 and GFP-ORP1L $\triangle O R D$, as detected by HA ${ }^{10} \mathrm{~nm}$ and GFP15 $\mathrm{nm}$ gold antibodies. Insets show ORP1L labelling in the membrane contact site between ER and autophagosome. The membranes of the ER are depicted in the bottom inset. Scale bar, $50 \mathrm{~nm}$. (c) Three-colour super-resolution image of an autophagosomal vesicle labelled by LC3 (green), ORP1L (blue) and the ER protein VAP-A (red). Scale bar, $500 \mathrm{~nm}$ (d) MelJuSo cells cultured either in lipid depleted serum or control medium were fixed and stained for LC3 and ER marker Calnexin. Scale bar, $10 \mu \mathrm{m}$. Right panel: Manders coefficient for LC3 localization to the ER was calculated on at least 10 cells over three independent experiments. Bars indicate mean + s.d. Student's $t$-test statistical analysis $\left({ }^{\star \star \star} P<0.0001\right)$.

autophagic flux (Supplementary Fig. 5a,b), in agreement with others $^{4,45}$, we found that increased lysosomal cholesterol levels prevented autophagosome-LE/Ly fusion (positioning them in very close proximity, Supplementary Fig. 5a,b). Since cholesterol reduction affects many cellular processes, it could be that compensatory mechanisms are in place, or that the cholesterol reduction is not strong enough to observe an effect on autophagic flux. Yet, the observed block in fusion with ORP1LAORD suggests a role for endosomal cholesterol in autophagosome maturation.
ORP1L controls PLEKHM1/HOPS acquisition by RAB7-RILP. The observations that ORP1L blocks autophagosome-LE/Ly fusion, together with the preferential localization of ORP1L to Rab7-positive compartments, suggest that ORP1L likely affects fusion between autophagosomes and LE/Lys from the latter side. Recent work has implicated the Rab7 effector PLEKHM1 as pertinent to this fusion step ${ }^{17}$. To test a possible functional interplay between ORP1L and PLEKHM1, we co-expressed GFP-tagged PLEKHM1 with ORP1L and its mutants. Strikingly, ectopic expression of ORP1LAORD inhibited distribution of 
a

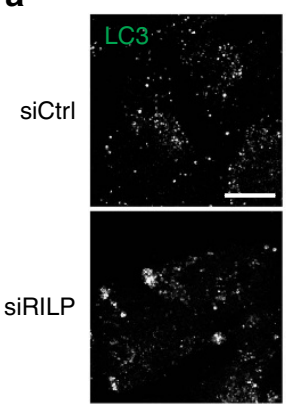

C

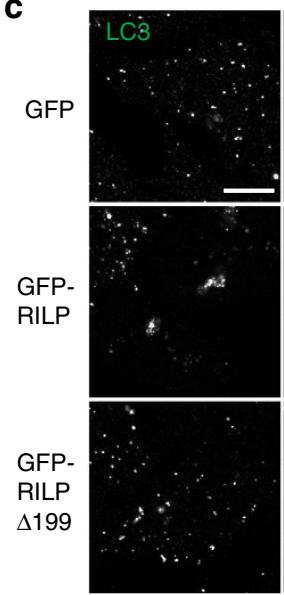

e
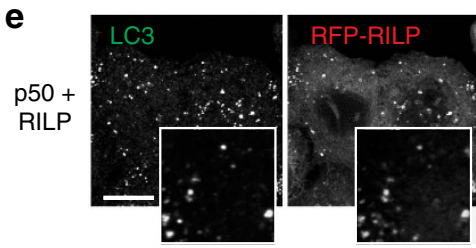

g

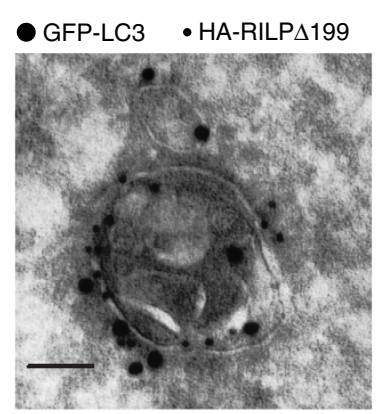

h
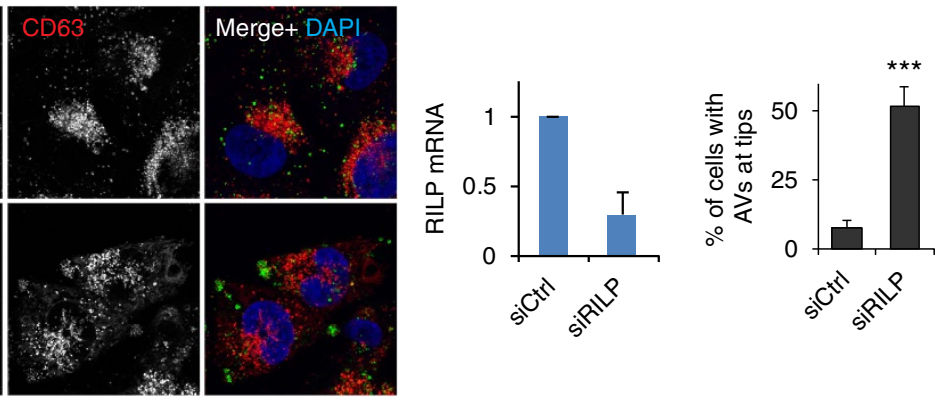

d

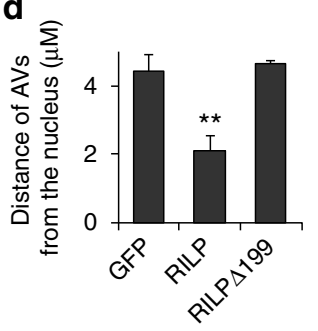

f
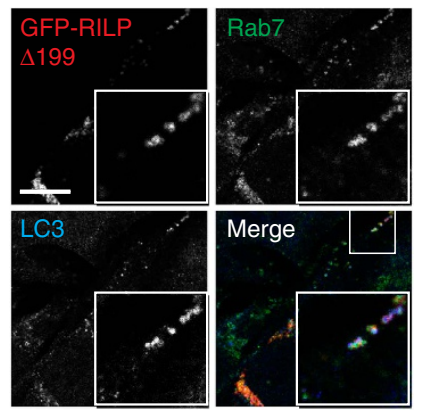

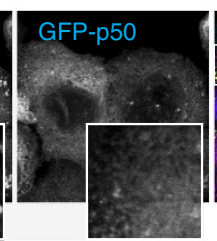

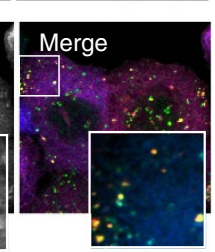
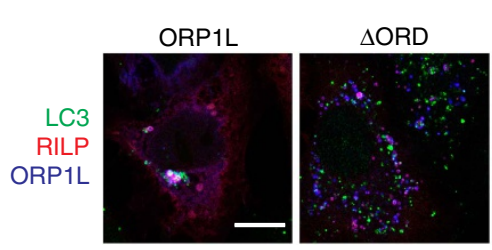

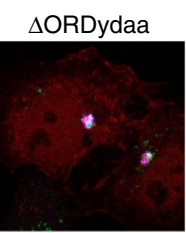

i

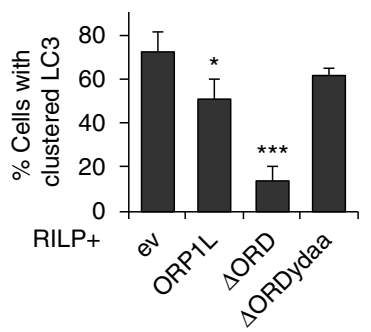

Figure 4 | ORP1L controls RILP mediated transport of late autophagosomes. (a) MelJuSo cells transfected with siCtrl or siRILP were stained for LC3, CD63 and DAPI. Scale bar, $10 \mu \mathrm{m}$. Right panel; silencing efficiency of RILP was determined by qRT-PCR, RILP signal was normalized to GAPDH. Bars indicate mean + s.d. of three experiments. (b) Quantification of cells with AVs located at the tips of cells from $\mathbf{a},>100$ cells per experiment were quantified, bars show mean + s.d. of three independent replicates. Means were compared using Student's $t$-test (c) HeLa cells transfected with the indicated constructs were fixed and stained for LC3 and CD63, as indicated. Scale bar, $10 \mu \mathrm{m}$. (d) Quantification of the average distance ( $\mu \mathrm{m}$ ) of AVs from the nucleus (by LasAF software) as shown in c, from $>10$ cells per experiment. Bars display mean + s.d. of three independent experiments, significance was calculated using Student's $t$-test. (e) HeLa cells transfected with the indicated constructs were stained for LC3. Scale bar, $10 \mu$ m (f) MelJuSo cells expressing GFP-RILP $\Delta 199$ were stained for Rab7 and LC3. Scale bar, $10 \mu \mathrm{m}$. (g) Immuno-electron micrograph of a HeLa cell expressing GFP-LC3 and HA-RILPA199, detected with 15 and $10 \mathrm{~nm}$ gold, respectively. Note the single membrane around the LC3-positive structure. Scale bar, $100 \mathrm{~nm}$ (h) HeLa cells expressing GFP-RILP and HAtagged ORP1L constructs were fixed and stained for HA and LC3. Scale bar, $10 \mu \mathrm{m}$. (i) Quantification of the number of cells with perinuclear accumulation of LC3-positive structures from $\mathbf{h}$. Quantified $>100$ cells per experiment. Bars indicate mean $+\mathbf{s}$.d. from independent triplicate experiments. Student's $t$-test statistical analysis. ${ }^{\star} P<0.05,{ }^{\star \star} P<0.01,{ }^{\star \star \star} P<0.001$. ev, empty vector control.

PLEKHM1 to Rab7, yielding cytosolic PLEKHM1 instead (Fig. 6a). Mutating the VAP-A interacting FFAT motif of ORP1L restored acquisition of PLEKHM1 by Rab7, suggesting that ORP1L does not simply compete with PLEKHM1 for Rab7-binding space, but rather actively informs PLEKHM1 recruitment in a manner dependent on the association with the ER protein VAP-A.

To mediate fusion, PLEKHM1 binds the HOPS complex, a multiprotein complex organizing the SNARE proteins for 
a

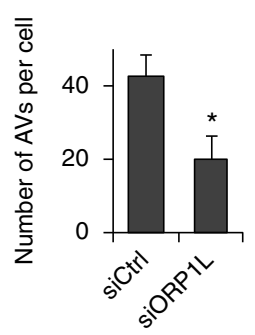

b

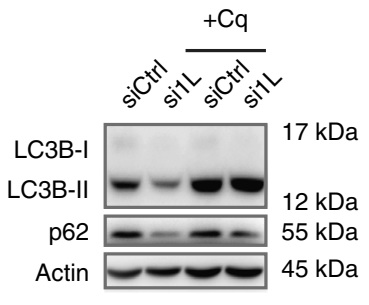

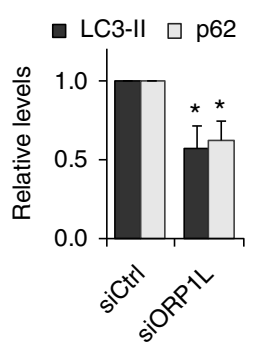

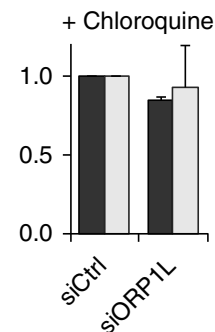

C

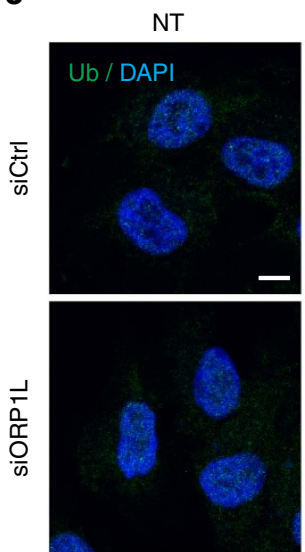

d
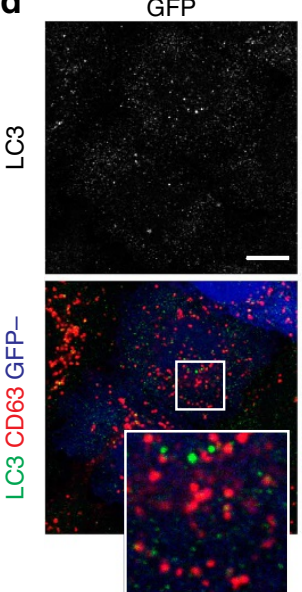

e

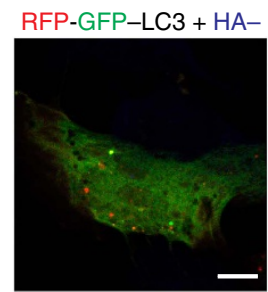

Puro $2 \mathrm{~h}$

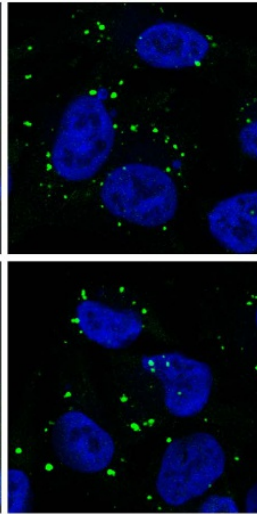

ORP1L

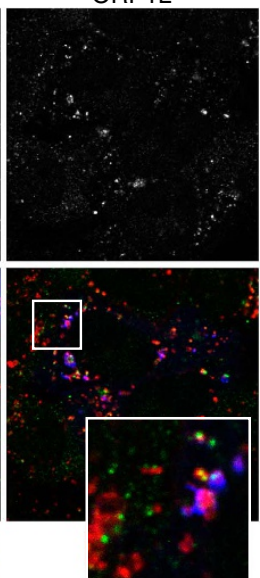

ORP1L

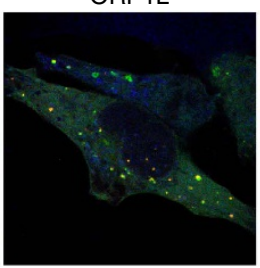

$2 \mathrm{~h}+$ washout $6 \mathrm{~h}$
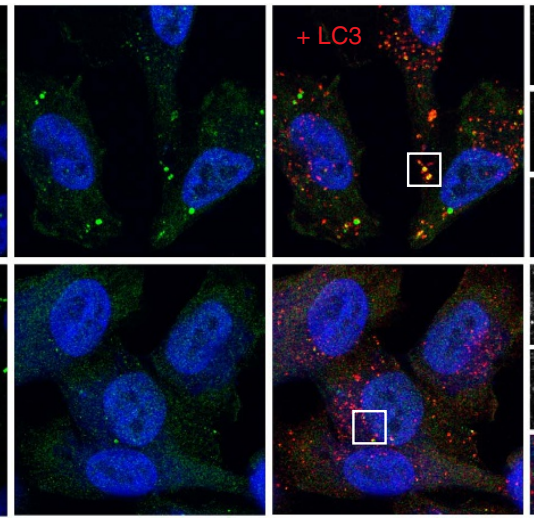

ORP1L $\triangle$ ORDydaa
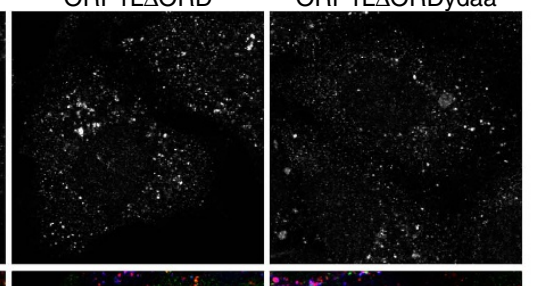

Early
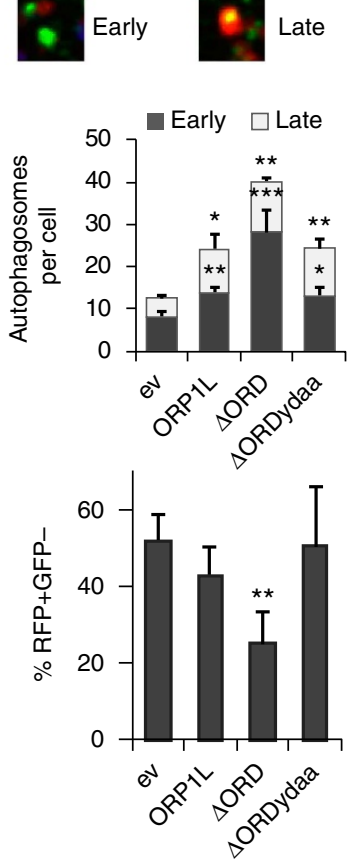

Figure 5 I ORP1L controls autophagosome maturation. (a) Quantification of the number of autophagosomes in MelJuSo cells transfected with siCtrl or siORP1L, from Fig. 2a. Number of LC3 structures was quantified in $>10$ cells per experiment, bars show mean + s.d. from three independent replicates. Means were compared using Student's t-test. (b) MelJuSo cells transfected with siCtrl or siORP1L were either or not exposed to $50 \mu \mathrm{M}$ chloroquine for $4 \mathrm{~h}$, lysed and analysed by SDS-PAGE and western blotting for p62, LC3B and actin. Right panels, quantification of signals for p62 and LC3B-II, corrected for loading (actin) and normalized to siCtrl, untreated (left) and chloroquine treated (right). Bars correspond to mean + s.d. of three experiments, significance was calculated using Student's $t$-test. (c) MelJuSo cells transfected with siCtrl or siORP1L were left untreated (NT) or treated with $4 \mu \mathrm{gl}^{-1}$ puromycin for $2 \mathrm{~h}$. Then, cells were washed and left to recover for the indicated time points before fixation and staining for ubiquitin (Ub), LC3 and DAPI. Scale bar, $10 \mu \mathrm{m}$. Right panel: quantification of percentage of cells with cytosolic Ub aggregates, $>100$ cells per experiment and bars represent mean + s.d. of three independent experiments. Means were compared using Student's t-test. (d) HeLa cells expressing the indicated GFP-tagged constructs were fixed and stained for LC3, CD63 and DAPI. Scale bar, $10 \mu \mathrm{m}$. Right panel: quantification of $\mathrm{LC3}+\mathrm{CD} 63$ - (early, green) and LC3 + CD63 + (late, yellow) autophagosomes detected in cells with indicated expressed proteins. Quantification of at least 10 cells per experiment, bars represent mean + s.d. of three independent experiments. Significance calculated using Student's $t$-test. (e) MelJuSo cells expressing the indicated HA-tagged constructs in combination with the mRFP-GFP-LC3 tandem construct were starved for $4 \mathrm{~h}$, fixed and stained for HA. Scale bar, $10 \mu \mathrm{m}$. Right panel: quantification of percentage of RFP-only LC3 structures relative to total LC3 labelled structures. Quantification of at least 10 cells per experiment is shown. Bars indicate mean + s.d. from independent triplicate experiments, statistical analysis using Student's $t$-test. ${ }^{\star} P<0.05,{ }^{\star \star} P<0.01,{ }^{\star \star \star} P<0.001$. ev, empty vector. 
a

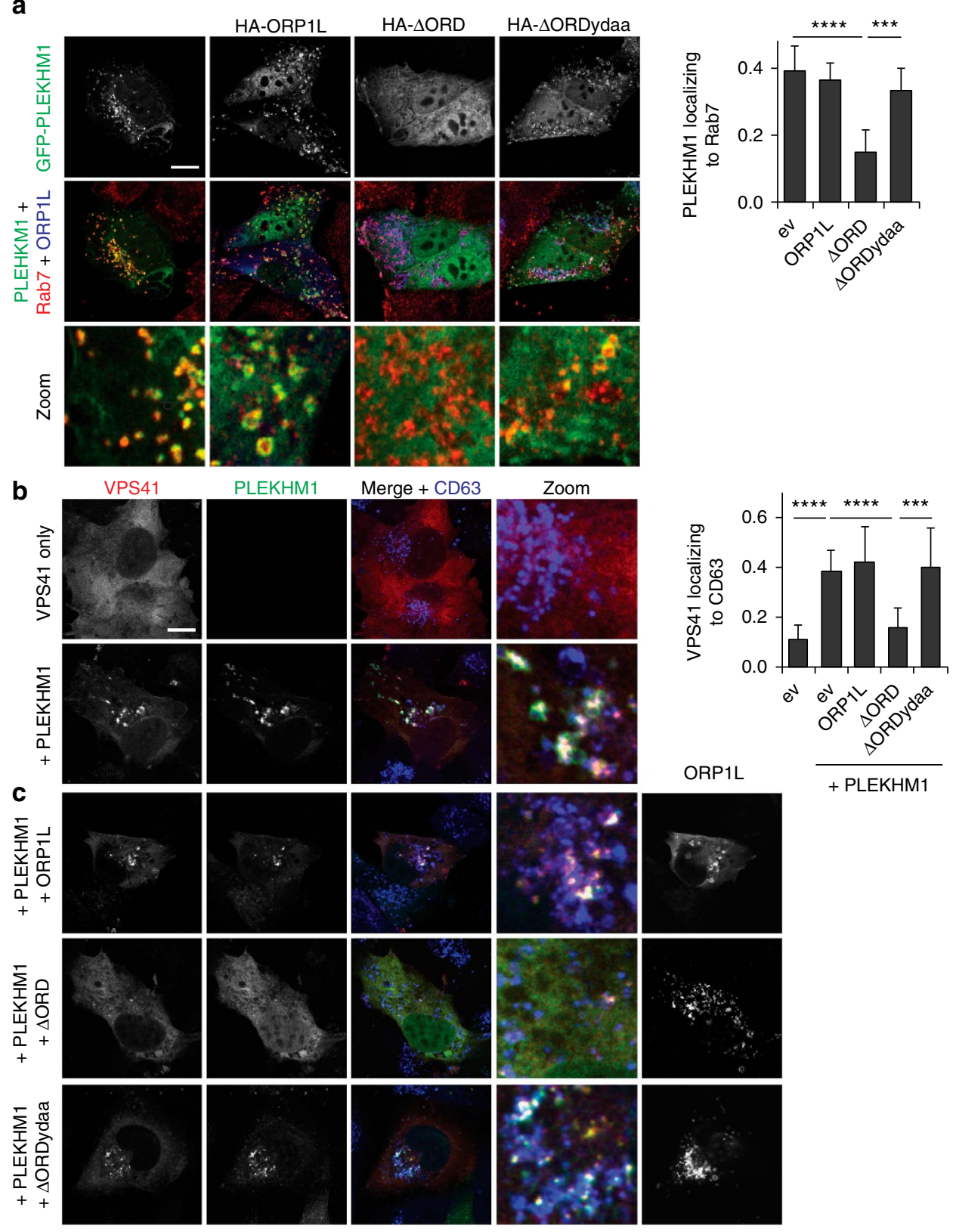

Figure 6 | ORP1L regulates recruitment of PLEKHM1 and HOPS to Rab7 positive vesicles. (a) MelJuSo cells expressing GFP-tagged PLEKHM1 and the indicated HA-tagged ORP1L mutants were fixed and stained for Rab7. Scale bar, $10 \mu \mathrm{m}$. Right panel: Manders coefficient for PLEKHM1 localization to Rab7, as determined for $>10$ cells in three experiments. Bars represent mean $+\mathbf{s}$.d. and significance calculated using Student's $t$-test. (b,c) MelJuSo cells were transfected with GFP-tagged PLEKHM1, mRFP-VPS41 and the indicated HA-tagged ORP1L mutants and stained for CD63. Manders coefficient for VPS41 localization to CD63 was determined for $>10$ cells in three experiments. Scale bar, $10 \mu \mathrm{m}$. Bars display mean $+\mathrm{s} . \mathrm{d}$., means were compared using Student's t-test. ${ }^{\star \star \star} P<0.001,{ }^{\star \star \star \star} P<0.0001$. ev, empty vector.

fusion $^{46}$, likely through a direct interaction with HOPS subunit VPS39 (ref. 17). Whether this interaction drives recruitment of the entire HOPS complex to Rab7 is unknown. To test this, we expressed several HOPS subunits in combination with PLEKHM1. While all subunits tested were cytosolic when expressed on their own, co-expression of PLEKHM1 relocalized both VPS39 and VSPS41 to LEs/Lys (Fig. 6b; Supplementary Fig. 6a). No recruitment was observed for an alternate HOPS complex member, VPS33b (refs 16,20; Supplementary Fig. 6b), implying that PLEKHM1 specifically recruits the HOPS core complex to Rab7. Introduction of ORP1L $\Delta$ ORD blocked membrane distribution of both VPS39 and VPS41, as well as PLEKHM1 in a manner dependent on the FFAT motif (Fig. 6c;
Supplementary Fig. 6a). At the same time, ORP1L $\Delta$ ORD had no effect on localization of fusion factor VAMP8 (Supplementary Fig. 6c). These data support the premise that ORP1L-mediated ER-contact sites orchestrate tethering of autophagosomes with LE/Ly by modulating recruitment of the autophagic effector PLEKHM1, and subsequently that of HOPS, to Rab7.

Besides PLEKHM1, also RILP can recruit the HOPS complex to Rab7, through a direct interaction with VPS41 (refs 15,19). This begs the question whether both Rab7 effectors-RILP and PLEKHM1 - mediate fusion of different vesicular subpopulations, or rather cooperate to recruit HOPS, the latter possibility supported by the requirement for both adaptors in ensuring efficient access of endocytosed EGFR to the LE/Ly 
compartment ${ }^{17,47}$. RILP and PLEKHM1 robustly co-localized on the

same vesicles (Fig. 7a; Supplementary Fig. 7a), and silencing RILP relegated PLEKHM1 to the cytosol (Fig. 7b), implying a functional interplay between the two effectors. The latter was not related to the HOPS recruitment capacity of RILP, since the RILP $\Delta 199$ mutant (which fails to recruit HOPS ${ }^{15}$ ) did not affect endosomal membrane targeting of PLEKHM1 (Fig. 7a; Supplementary Fig. 7a). Furthermore, expression of constitutively active Rab7(Q67L) restored membrane targeting of PLEKHM1 (Supplementary Fig. 7b), indicating that the phenotype is likely a result of an altered activated Rab7 state, as observed previously for effector silencing or overexpression ${ }^{18,28}$. While in the presence of RILPA199, PLEKHM1 was still able to recruit
VPS39 (Fig. 7c; Supplementary Fig 7c), it poorly recruited VPS41 to endosomes (Fig. 7c). Co-immunoprecipitation experiments confirmed that the interaction between PLEKHM1 and VPS41 was attenuated by RILP $\Delta 199$ (Fig. 7d), implying that functional RILP is required for PLEKHM1 to mobilize VPS41 and thus the entire HOPS complex. RILP and PLEKHM1 physically associated with each other, which was strongly reduced by using RILP $\Delta 199$, and more efficient when co-expressing VPS41 (Fig. 7e), suggesting that RILP and PLEKHM1 form a complex via HOPS. Together, these observations suggest that the HOPS complex is jointly recruited to LE/Lys by the Rab7 effectors PLEKHM1 and RILP, where PLEKHM1 binds to HOPS subunit VPS39 and RILP to VPS41. Whereas RILP remains associated to Rab7 following formation of ORP1L-VAP-A contact sites by
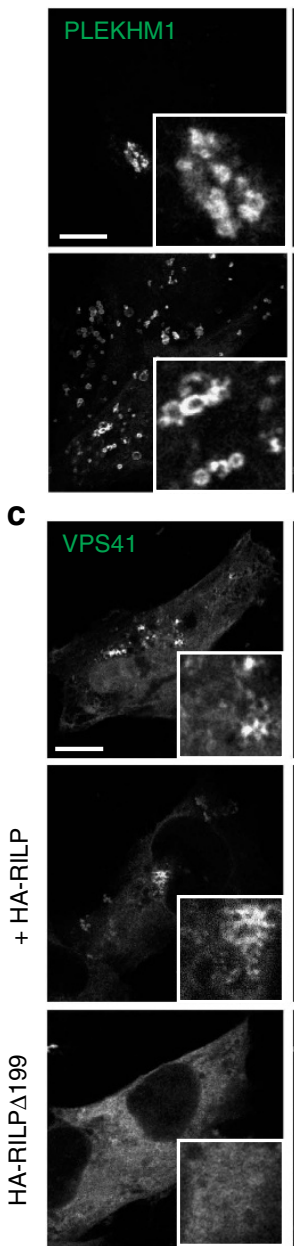
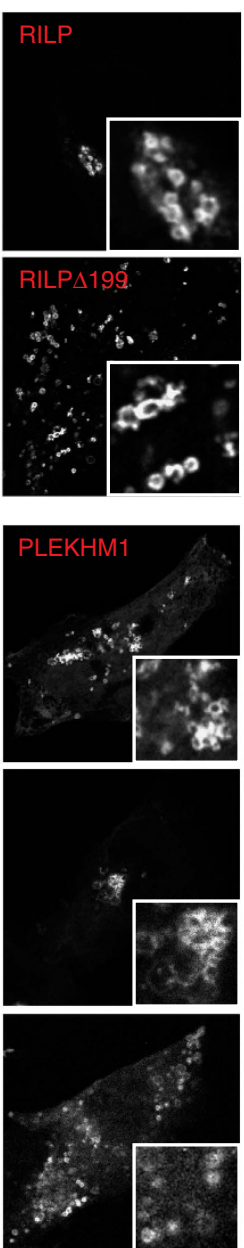
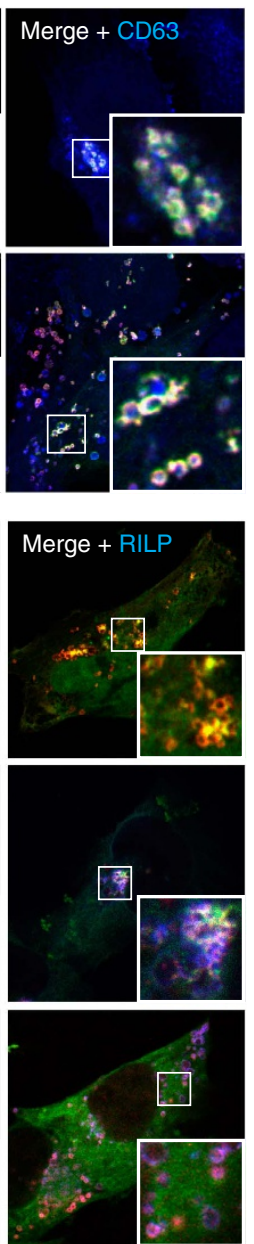

b
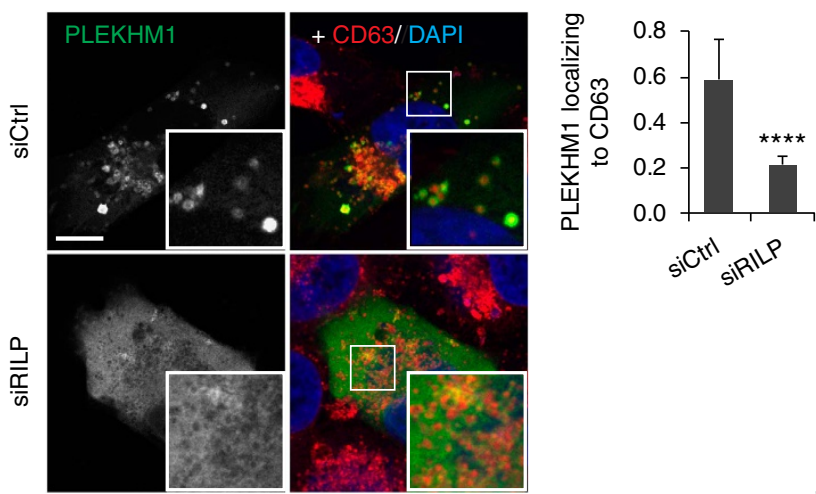

— VPS39:PLEKHM1 $\square$ VPS41:PLEKHM1

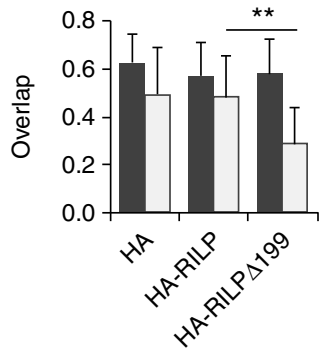

d $\mathrm{HA}$ HA-VPS41 Flag-PLEKHM1

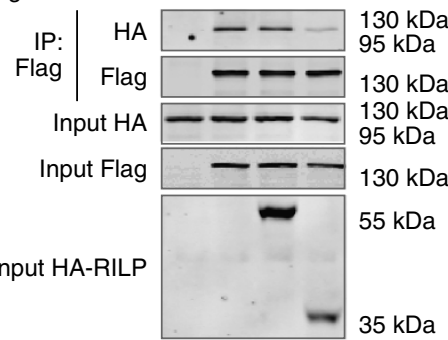

GFP-

Flag-PLEKHM1

HA-VPS41

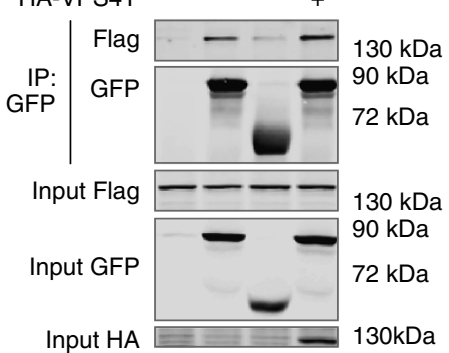

Figure 7 | Rab7 effectors PLEKHM1 and RILP cooperate in HOPS complex recruitment. (a) MelJuSo cells were transfected with GFP-PLEKHM1 and HA-RILP or HA-RILPA199 and stained for CD63, as indicated. Scale bar, $10 \mu \mathrm{m}$. (b) MelJuSo cells silenced for siCtrl or siRilp were transfected with GFP-PLEKHM1 and stained for CD63. Manders coefficient for PLEKHM1 localization to CD63 was calculated for >10 cells over three experiments. Bars display mean + s.d., significance determined using Student's $t$-test. Scale bar, $10 \mu \mathrm{m}$. (c) MelJuSo cells were transfected with GFP-PLEKHM1 and mRFPVPS41, together with HA-RILP or HA-RILPA199 when indicated. Manders coefficient for VPS39 (Supplementary Fig. 6c) or VPS41 localization to PLEKHM1 was calculated for $>10$ cells in three individual experiments. Bars represent mean + s.d. and significance was calculated using Student's $t$-test. Scale bar, $10 \mu \mathrm{m}$. (d) Co-immunoprecipitation for Flag-PLEKHM1 and HA-VPS41 in the presence of HA-RILP or HA-RILPA199. HEK293T cells were transfected with the indicated constructs and immunoprecipitated for Flag. Bottom: quantification of co-immunoprecipitated HA-VPS41 signal corrected for input and normalized to control, $n=4$. Bars display mean + s.d., means were compared using Student's $t$-test. (e) Immunoprecipitation for GFP or GFP-tagged RILP constructs in HEK293T cells co-expressing Flag-PLEKHM1 with or without HA-VPS41, as indicated. ${ }^{\star} P<0.05,{ }^{\star \star} P<0.01,{ }^{\star \star \star} P<0.001,{ }^{\star \star \star \star} P<0.0001$. 
ORP1L $\triangle \mathrm{ORD}^{15}$, PLEKHM1 and the HOPS complex are released, obstructing fusion of autophagosomes with LEs/Lys (Fig. 8).

\section{Discussion}

Autophagic proteolysis of bulky cargoes acquired in the cytosol necessitates their entry into the endocytic system for degradation by lysosomal hydrolases. Endosomes present a challenging target, as they traverse different maturation stages, all while moving quickly between the peripheral and interior regions of the cell. How fusion and transport of autophagosomes are negotiated within this dynamic vesicle network in space and time is poorly understood. Here we show that maturing autophagosomes hijack late endosomal fusion and transport machinery to access the lysosomal compartment, and in doing so, become subject to endosome-centric regulatory mechanisms. Among these, we identify a negative regulator of autophagosome fusion with the $\mathrm{LE} / \mathrm{Ly}$ - which functions as a cholesterol sensor poised on receiving vesicles to either licence or refute autophagic progression.

Enclosure of cytosolic substrates within $\mathrm{LC}^{+}$doublemembranes gives rise to early autophagosomes, which mature by fusion with lysosomes or endosomes harbouring late characteristics. For fusion to take place, the autophagic membrane-bound SNARE protein STX17 (ref. 6) needs to contact the endosome-associated HOPS tethering complex ${ }^{13,14}$. On the LE/Ly side of the merger, the small GTPase Rab7 recruits its effectors PLEKHM1 and RILP, capable of respectively binding the VPS39 and VPS41 components of HOPS. Our data suggest that these effectors jointly recruit the same HOPS complex, which is analogous to yeast, where the Rab7 homologue YPT7 acquires HOPS by binding to both VPS39 and VPS41 (refs 48,49). Since RILP and PLEKHM1 bind the same motif on Rab7 (ref. 18), two Rab7 molecules probably associate to the two different effector proteins-RILP and PLEKHM1 - which associate to two subunits of one tethering HOPS complex.

In addition to PLEKHM1 and RILP, Rab7 recruits another effector, the cholesterol-sensor ORP1L, which binds Rab7 in the presence of RILP ${ }^{40}$ and engages directly with ER-located VAP-A. Genetic manipulation of underlying interaction determinants casts $\mathrm{AV} / \mathrm{LE}$ fusion as subordinate to complex formation between ORP1L and VAP-A. In support of this, we find that in the presence of ORP1L predisposed to binding VAP-A, PLEKHM1 becomes relegated to the cytosol, while RILP remains associated to Rab7 but releases HOPS. This results in diminished entry of $\mathrm{LC}^{+}$compartments into the acidified luminal LE environment and expansion of the now-arrested early autophagosomal repertoire. Given that ORP1L-mediated ER-contact sites are modulated by cholesterol or oxysterols as recognized by the ORD domain of ORP1L, cholesterol on the cytosolic leaflet of Rab7-ORP1L compartments could control the autophagic flux. While the effects of the different ORP1L mutants on the autophagic flux revealed a clear and interpretable effect, this was less so for lipid manipulation of cells. This could be due to the milder nature of cholesterol manipulation, the multitude of other pathways that are affected by cholesterol, or another unknown factor controlling ORP1L contact site formation.

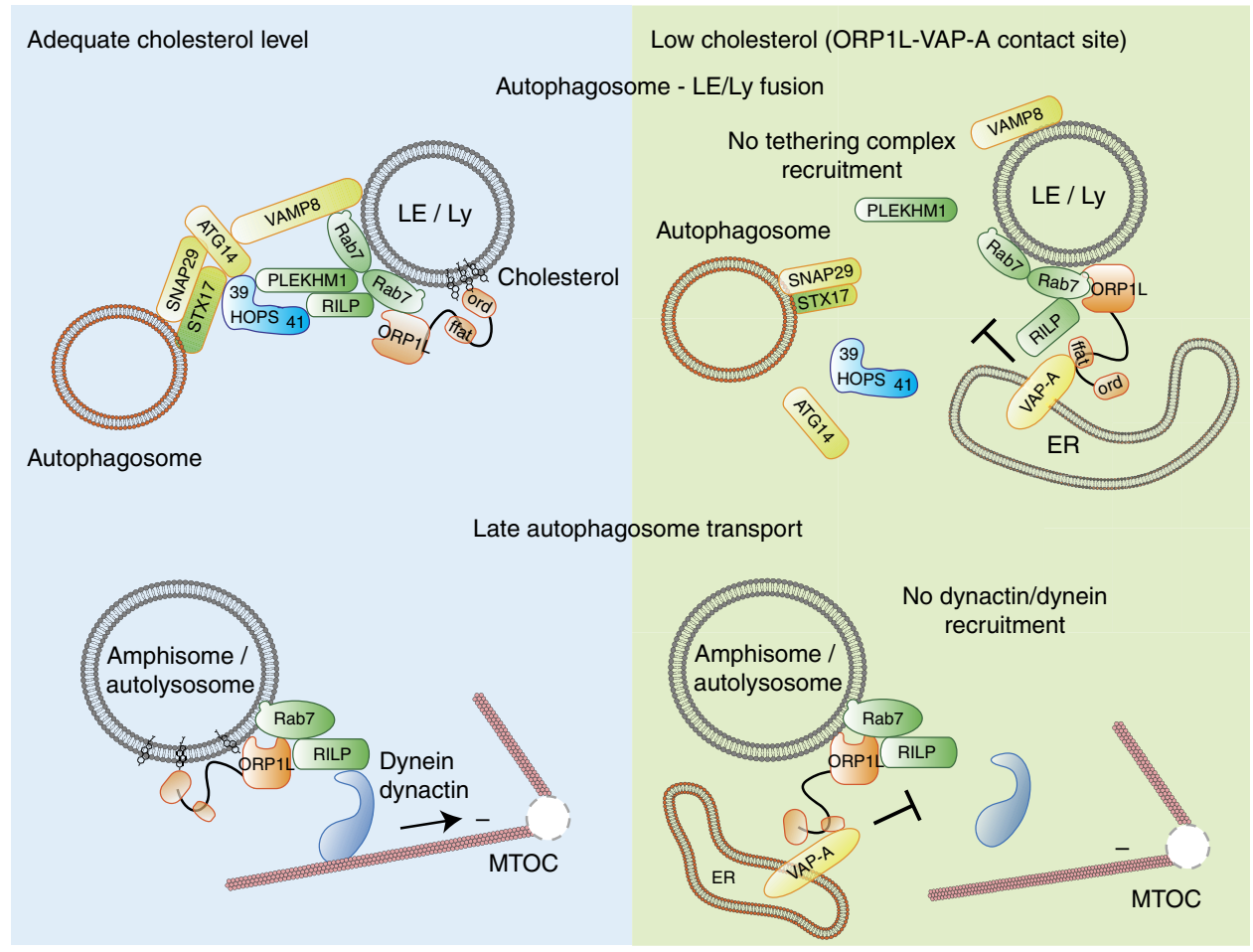

Figure 8 | Model for ORP1L-mediated control of autophagosome-LE/Ly fusion and late autophagosome transport. SNARE proteins for fusion are depicted in yellow, tethering factors in green and the HOPS complex in blue. Tethering of autophagosomes with LE is orchestrated by two Rab7 molecules binding to two effectors PLEKHM1 and RILP, that respectively bind the VPS39 and VPS41 subunits of the HOPS tethering complex. From the autophagosome side, the SNARE Syntaxin 17 binds HOPS and mediates fusion together with the SNAREs SNAP29 and VAMP8, and ATG14. Under lowcholesterol conditions, the ORD domain of ORP1L is released from the membrane, exposing the FFAT motif that interacts with ER protein VAP-A. This membrane contact site prevents recruitment of PLEKHM1 and HOPS to Rab7-RILP, and thereby tethering and fusion. Once fusion does occur, RILP recruits dynactin-dynein to amphisomes to promote minus-end transport. Here ORP1L can also form a contact site with VAP-A, which prevents dynactin recruitment and blocks minus-end transport. Thus, conformational changes in the cholesterol-sensor ORP1L time the fusion and transport of $A V s$. $L E$, late endosome; Ly, lysosome; 39, VPS39; 41, VPS41. 
However, our data with the ORP1L mutants suggest that cholesterol is instrumental for the autophagic flux, and demonstrate that the interplay between ORP1L and VAP-A determines whether a given AV/LE encounter will result in fusion, and by extension maturation of the autophagosome en route to proteolysis.

Following AV/LE fusion, the resulting amphisome needs to reach the lysosome to fulfil proteolysis of its contents. A large share of motor-based transport of autophagic vesicles is accounted for by amphisomes $21,24,50$, and several transport adaptors, including dynein intermediate chain-interacting proteins Snapin ${ }^{24,51}$ and Huntingtin ${ }^{52,53}$ are common between amphisomes and LEs. We demonstrate that $\mathrm{LC}^{+}{ }^{+}$amphisomes retain both transport and regulatory determinants of its parent LE, all converging onto the small GTPase Rab7. On RILP engagement with amphisomes, the dynein-dynactin complex can be recruited for minus-end-directed transport towards lysosomes, typically congregating near the microtubule organizing center. Concomitant association of ORP1L to the Rab7-RILP complex adds an additional layer of regulation, involving a novel membrane contact site between amphisomes and the ER. Superresolution fluorescence imaging and immuno-EM revealed ORP1L on single-membrane-enclosed $\mathrm{LC}^{+}$amphisomes, juxtaposed against VAP-A on the ER, indicating an interaction occurring in trans rather than resulting from possible ERGICderived VAP-A spillover into nascent autophagosomes ${ }^{54}$. Formation of the amphisome-ER contact site is cholesterol dependent, advocating that via ORP1L, cholesterol can toggle decisions regarding transport of amphisomes. Why cholesterol is used to mark this step in autophagosome maturation is unclear. Possibly ORP1L confines the active transport machinery to defined regions on a vesicle, cholesterol-enriched subdomains. If so, decisions regarding transport are restricted to a few locations on a vesicle, so-called transport nodes, which potentially simplifies regulation of bidirectional transport. In support of this theory, it was recently shown that on phagosomes Rab7 and the dynein motor are recruited specifically to cholesterol-enriched subdomains to control minus-end transport ${ }^{55}$. Interestingly, plusend transport of amphisomes depends on $\mathrm{PI}(3) \mathrm{P}$ binding by FYCO1 (ref. 31), suggesting a broader role for lipids in the regulation of amphisome transport. ORP family members have been demonstrated to also bind phosphoinositides within their ORD domain ${ }^{56-58}$, which may also apply for the ORD domain of ORP1L. The physiological role for transport regulation by cholesterol or other putative ligands of ORP1L is unclear, but it is possible that it functions as a timer, where older amphisomes can be recognized from a distinct lipid composition of their cytosolic leaflet, leading to minus-end transport and subsequent fusion with the lysosome. On top of the transport regulating ORP1L-VAP-A contact sites, we also observed cholesterol- and VAP-A-dependent but ORP1L-independent AV/ER contact sites. These could be facilitated by other ORP family members harbouring the FFAT motif, or afforded by the divergent cholesterol-binding proteins STARD3 or STARD3NL ${ }^{38,59,60}$, and will be subject for further study. Thus, via ORP1L, the ER and lipids in the limiting membranes of endosomes regulate late autophagosome transport, illustrating its complexity and intercompartmental control.

In addition to their role in autophagy induction through the regulation of mTOR activity ${ }^{30,61}$, our data implicate LE/Ly as potent drivers of autophagic flux, capable of imposing restrictions on acceptance of autophagic vesicles into its fold. Fusion is thus not simply imposed by the autophagosome, but is also subject to licence by the LE. This mechanism can be exploited on the individual endosome basis and activated on a specific set of LE/Ly, for example, melanosomes or exocytic LEs/Lys, to prevent incoming cargo. Alternatively, ORP1L could serve as a quality control switch on LE/Ly to prevent incoming traffic when the endosome is not equipped to handle additional cargo, for example, just after acquiring autophagosomal cargo. Following autophagic lysosome reformation ${ }^{62}$, revitalized endosomes could inactivate ORP1L to take on a new degradation cycle. Given that starvation enhances $\mathrm{AV} / \mathrm{LE}$ fusion ${ }^{63}$, it will be interesting to investigate a potential interplay between mTOR signalling and ORP1L activity.

Collectively, our data suggest (Fig. 8) that the cholesterol sensor ORP1L regulates AV/LE fusion, and attenuates recruitment of PLEKHM1 and HOPS to Rab7-RILP complexes. Rab7 effectors PLEKHM1 and RILP then jointly attract HOPS for fusion. Following fusion, the resulting amphisome utilizes Rab7-RILP-associated dynein motor machinery for minus-enddirected transport, which is also under control of the very same ORP1L, through a novel type of MCS engaging late autophagosomal structures with the ER. These data provide a molecular basis for the potential role of a major dietary lipid, cholesterol, in controlling autophagosome transport and degradation of its content in lysosomal compartments ${ }^{64,65}$.

\section{Methods}

Cell culture and constructs. MelJuSo cells were cultured in IMDM supplemented with 10\% FCS, HeLa and HEK 293 T cells in DMEM with 10\% FCS. All cells were checked for contamination by other cells (using morphological analysis and surface marker expression) and were regularly tested for mycoplasma contamination. Constructs for expression of ORP1L, Rab7, RILP, p50, VPS39, VPS41, VPS33b, VAMP8 and VAP-A were described previously ${ }^{15,33}$. HA-ORP1L and mutants were obtained by swapping GFP in the GFP-C1 vector for a 2xHA tag.

ORP1L $\Delta$ ORDydaa was generated by mutating the residues Y477 and D478 into alanines using the primers: $5^{\prime}$-gaggacgagttcgctgcggcgctgtcagattccg- $3^{\prime}$ and

$5^{\prime}$-tctgacagcgccgcagcgaactcgtcctcgctaag- $3^{\prime}$. Both residues were mutated because a minor interaction with VAP-A was still observed in the D478A single amino acid mutant. PLEKHM1-GFP was a kind gift from Wim van Hul (Department of Medical Genetics, University of Antwerp, Belgium) ${ }^{66}$ and was recloned into 2Flag-N1 using XhoI and HindIII. mRFP-mGFP-LC3 was generated by cloning mGFP and LC3 into an mRFP-C1 vector, mCherry-LC3 by cloning LC3 into an mCherry- $\mathrm{C} 1$ vector. All constructs were sequence verified.

Reagents and antibodies. Lipid depletion was carried out by incubating cells in DMEM/IMDM supplemented with 5\% delipidized bovine serum (Pel-Freez Biologicals) in the presence of $50 \mu \mathrm{M}$ lovastatin (Millipore) to inhibit cholesterol production and $230 \mu \mathrm{M}$ Mevalonate (Sigma-Aldrich) to supply essential non-sterol isoprenoids. For endosomal cholesterol accumulation, cells were treated with $3 \mu \mathrm{g} \mathrm{ml}^{-1} \mathrm{U}-18666 \mathrm{~A}$ (Cayman Chemicals), for lysosomal staining Lysotracker green (Molecular Probes) was used. Starvation, used for the mRFP-mGFP-LC3 experiment, was performed by culturing cells in EBSS (ThermoScientific) for the indicated time. Puromycin (Gibco) was used at $4 \mu \mathrm{g} \mathrm{ml}^{-1}$. Antibodies used: rabbit anti-LC3B (Novus Biologicals NB100-2220, used for all experiments except costaining with endogenous Rab7, microscopy 1:400, western blot 1:1,000), mouse anti-LC3B (Cosmo Bio LC3-1703, only used for co-staining with Rab7, 1:100), rabbit anti-ORP1L $(1: 1,000)$, rabbit anti-GFP (1:1,000), mouse anti-CD63 (1:500) and rabbit anti-CD63 (1:100) (all described in ref. 33), rabbit anti-PDI (1:10, as described in ref. 67), mouse anti-HA (Covance 16B12, 1:1,000), rat anti-HA 3F10 (Roche, 1:200), mouse anti-p62 D3 (1:500), goat anti-calnexin C-20 (1:40), goat anti-VAP-A K15 (microscopy 1:40, western blot 1:500), mouse anti-Ubiquitin P4D1 (1:50) (all Santa Cruz), rabbit anti-Rab7 D95F2 (Cell Signaling Technologies, 1:100), and mouse anti-p150 glued (BD Biosciences 610473, 1:100), rabbit anti-Flag F7425 and mouse anti-Flag M2 (Sigma, 1:1,000). Secondary antibodies (goat anti-mouse Alexa 405/488/568/647, goat anti-rabbit Alexa 488/568/647, donkey anti-goat Alexa 488/568, donkey anti-mouse Alexa 488/555/647 and donkey anti-rabbit Alexa 488/647) were purchased from Life Technologies and donkey anti-rat CF568 from Biotium (all 1:200).

Transfections. For expression studies, MelJuSo and HeLa cells were transfected using Effectene (Qiagen) according to the manufacturer's instructions. Cells were transfected 1 day before fixation or lysis. For siRNA-mediated depletion, cells were reverse transfected with DharmaFECT transfection reagent \#1 and $50 \mathrm{nM}$ siRNA (catalogue numbers: siCtrl: D00120613-20, siORP1L: D008350-(01,05,18,19), siVAP-A: D021382-(01,02,03,04), siRILP D008787-(01,02,03,04) of the Human siGenome SMARTpool, Dharmacon) according to the manufacturer's protocol Briefly, siRNAs and DharmaFECT were mixed and incubated for $20 \mathrm{~min}$ in a 
culture well, after which cells were added and left to adhere (reverse transfection). Three days later, cells were fixed and stained or lysed for biochemical analysis.

Co-immunoprecipitation and western blotting. For co-immunoprecipitation experiments, cells were lysed in lysis buffer $(0.5 \% \mathrm{NP}-40,5 \%$ glycerol, $150 \mathrm{mM} \mathrm{NaCl}$, $50 \mathrm{mM}$ Tris- $\mathrm{HCl} \mathrm{pH} 8.0,5 \mathrm{mM} \mathrm{MgCl}$ supplemented with complete EDTA-free Protease Inhibitor Cocktail (Roche)) and cleared by centrifugation. Lysates were incubated with GFP-Trap beads (Chromotek) or protein G-Sepharose 4 Fast Flow resin with the indicated antibodies and after incubation washed extensively with lysis buffer before addition of sample buffer ( $2 \%$ SDS, $10 \%$ glycerol, $5 \% \beta$-mercaptoethanol, $60 \mathrm{mM}$ Tris- $\mathrm{HCl} \mathrm{pH} 6.8$ and $0.01 \%$ bromophenol blue).

For whole cell lysate analyses, cells were lysed directly in sample buffer. Proteins were separated by SDS-PAGE (polyacrylamide gel electrophoresis) and transferred to western blot filters. Blocking of the filter and antibody incubations were done in PBS supplemented with $0.1(\mathrm{v} / \mathrm{v}) \%$ Tween and $5 \%(\mathrm{w} / \mathrm{v})$ milk powder. Blots were imaged using the Odyssey Imaging System (LI-COR) or ChemiDoc (Bio-Rad). Uncropped western blot images are presented in Supplementary Fig. 8.

CDNA synthesis and qPCR. RNA isolation, complementary DNA synthesis and quantitative PCR with reverse transcription were performed according to the manufacturer's (Roche) instructions. Signal was normalized to GAPDH and calculated using the Pfaffl formula. Primers used for detection of GAPDH and RILP were: GAPDH fw: $5^{\prime}$-TGTTGCCATCAATGACCCCTT-3', GAPDH rv: 5'-CTCCACGACGTACTCAGCG-3', RILP fw: $5^{\prime}$-AAGCAGCGGAAGAAGA TCAA-3', RILP rv: 5'-TTGTCATCGGAGAGCAGGAT-3'

Confocal microscopy. Cells were seeded on coverslips, transfected $18 \mathrm{~h}$ later and treated as indicated. Twenty-four hours later, cells were fixed in 3.7\% formaldehyde for $10 \mathrm{~min}$ and permeabilized in ice-cold methanol for $2 \mathrm{~min}$. Staining was performed with the antibodies mentioned above and 4,6-diamidino-2-phenylindole (Invitrogen) to stain DNA or Filipin (Cayman Chemicals) to detect cholesterol. Images were acquired using a Leica TCS SP5 confocal microscope (Leica

Microsystems, Wetzlar, Germany) at $\times 63$ magnification. For live-cell imaging, the microscope was equipped with a climate control chamber and cells were imaged for 8 min with $\sim 5$ frames per minute. Images were quantified using Image J plugin Jacob for Mander's coefficient calculations or FIJI's Trackmate plugin for live-cell imaging and processed using Adobe Photoshop and Illustrator.

Cryo-immunoelectron microscopy. Transfected HeLa cells were fixed for $2 \mathrm{~h}$ in $2 \%$ paraformaldehyde $+0,2 \%$ glutaraldehyde in $0.1 \mathrm{M}$ PHEM buffer $(60 \mathrm{mM}$ PIPES, $25 \mathrm{mM}$ HEPES, $2 \mathrm{mM} \mathrm{MgCl}_{2}, 10 \mathrm{mM}$ EGTA, pH 6.9) and then processed for ultrathin cryosectioning. Briefly, 50-nm cryosections were cut at $-120^{\circ} \mathrm{C}$ using diamond knives in a cryoultramicrotome (Leica Aktiengesellschaft, Vienna, Austria) and transferred with a mixture of sucrose and methylcellulose onto formvar-coated copper grids. The grids were placed on $35-\mathrm{mm}$ Petri dishes containing $2 \%$ gelatine. Ultrathin frozen sections were incubated at room temperature with primary antibody and then incubated with $10 \mathrm{~nm}$ protein A-conjugated colloidal gold (EM Lab, Utrecht University, The Netherlands) as described ${ }^{68}$. For double labelling, the sections were first washed and then fixed for another $10 \mathrm{~min}$ in $1 \%$ glutaraldehyde, blocked and incubated with the second primary antibody and $15 \mathrm{~nm}$ gold-labelled protein A. The sections were embedded in a mixture of methylcellulose and uranyl acetate and examined with a Philips CM10 electron microscope (FEI, Eindhoven, The Netherlands).

Super-resolution microscopy. Super-resolution microscopy was performed with a Leica SR GSD microscope (Leica Microsystems, Wetzlar, Germany) mounted on a Sumo Stage (\#11888963) for drift-free imaging. Collection of images was done with an EMCCD Andor iXon camera (Andor Technology, Belfast, UK) and an oil immersion objective (HCX PL Apo 100X, NA 1.47). Laser characteristics were $405 \mathrm{~nm} / 30 \mathrm{~mW}, 488 \mathrm{~nm} / 300 \mathrm{~mW}$ and $647 \mathrm{~nm} / 500 \mathrm{~mW}$, with the $405-\mathrm{nm}$ laser used for back pumping and the others for wide field/TIRF imaging. Ultra clean coverslips (cleaned and washed with base and acid overnight) were used for imaging. The number of recorded frames was variable between 10,000 to 50,000 , with a frame rate of $100 \mathrm{~Hz}$. The data sets were analysed with the Thunder Storm analysis module $e^{69}$ and images were reconstructed with a detection threshold of 70 photons, sub pixel localization of molecules and uncertainty correction, with a pixel size of $10 \mathrm{~nm}$.

Statistical analysis and experimental set-up. All experiments shown in the paper are performed independently at least three times and images are representative of at least 10 cells viewed per individual experiment. For co-localization quantification, numerical values of individual cells were used for calculations, while for population quantifications, the averages per experiment were used. Statistical significance was calculated using an unpaired Student's $t$-test, except for normalized signals, for which a paired $t$-test was done. Statistical values are as following: ${ }^{\star} P<0.05,{ }^{* *} P<0.01,{ }^{* * *} P<0.001,{ }^{* * * *} P<0.0001$.
Data availability. All data supporting the findings in this study are included in the article, either in the main figures or the supplementary information files.

\section{References}

1. Yang, S. et al. Pancreatic cancers require autophagy for tumor growth. Genes Dev. 25, 717-729 (2011).

2. Blum, R. \& Kloog, Y. Metabolism addiction in pancreatic cancer. Cell Death Dis. 5, e1065 (2014).

3. Rubinsztein, D. C., Bento, C. F. \& Deretic, V. Therapeutic targeting of autophagy in neurodegenerative and infectious diseases. J. Exp. Med. 212, 979-990 (2015).

4. Gordon, P. B. \& Seglen, P. O. Prelysosomal convergence of autophagic and endocytic pathways. Biochem. Biophys. Res. Commun. 151, 40-47 (1988).

5. Diao, J. et al. ATG14 promotes membrane tethering and fusion of autophagosomes to endolysosomes. Nature 520, 563-566 (2015).

6. Itakura, E., Kishi-Itakura, C. \& Mizushima, N. The hairpin-type tail-anchored SNARE syntaxin 17 targets to autophagosomes for fusion with endosomes/ lysosomes. Cell 151, 1256-1269 (2012).

7. Takats, S. et al. Autophagosomal Syntaxin17-dependent lysosomal degradation maintains neuronal function in Drosophila. J. Cell. Biol. 201, 531-539 (2013).

8. Shen, H. M. \& Mizushima, N. At the end of the autophagic road: an emerging understanding of lysosomal functions in autophagy. Trends Biochem. Sci. 39, 61-71 (2014)

9. Gutierrez, M. G., Munafo, D. B., Beron, W. \& Colombo, M. I. Rab7 is required for the normal progression of the autophagic pathway in mammalian cells. J. Cell. Sci. 117, 2687-2697 (2004).

10. Jager, S. et al. Role for Rab7 in maturation of late autophagic vacuoles. J. Cell. Sci. 117, 4837-4848 (2004).

11. Ganley, I. G., Wong, P. M., Gammoh, N. \& Jiang, X. Distinct autophagosomallysosomal fusion mechanism revealed by thapsigargin-induced autophagy arrest. Mol. Cell 42, 731-743 (2011).

12. Kimura, S., Noda, T. \& Yoshimori, T. Dissection of the autophagosome maturation process by a novel reporter protein, tandem fluorescent-tagged LC3. Autophagy 3, 452-460 (2007).

13. Takats, S. et al. Interaction of the HOPS complex with Syntaxin 17 mediates autophagosome clearance in Drosophila. Mol. Biol. Cell 25, 1338-1354 (2014)

14. Jiang, P. et al. The HOPS complex mediates autophagosome-lysosome fusion through interaction with Syntaxin 17. Mol. Biol. Cell 25, 1327-1337 (2014).

15. van der Kant, R. et al. Late endosomal transport and tethering are coupled processes controlled by RILP and the cholesterol sensor ORP1L. J. Cell Sci. 126, 3462-3474 (2013).

16. Wartosch, L., Gunesdogan, U., Graham, S. C. \& Luzio, J. P. Recruitment of VPS33A to HOPS by VPS16 is required for lysosome fusion with endosomes and autophagosomes. Traffic 16, 727-742 (2015).

17. McEwan, D. G. et al. PLEKHM1 regulates autophagosome-lysosome fusion through HOPS complex and LC3/GABARAP proteins. Mol. Cell 57, 39-54 (2015).

18. McEwan, D. G. et al. PLEKHM1 regulates Salmonella-containing vacuole biogenesis and infection. Cell Host Microbe 17, 58-71 (2015).

19. Lin, X. et al. RILP interacts with HOPS complex via VPS41 subunit to regulate endocytic trafficking. Sci. Rep. 4, 7282 (2014).

20. van der Kant, R. et al. Characterization of the mammalian CORVET and HOPS complexes and their modular restructuring for endosome specificity. J. Biol. Chem. 290, 30280-30290 (2015)

21. Jahreiss, L., Menzies, F. M. \& Rubinsztein, D. C. The itinerary of autophagosomes: from peripheral formation to kiss-and-run fusion with lysosomes. Traffic 9, 574-587 (2008).

22. Kimura, S., Noda, T. \& Yoshimori, T. Dynein-dependent movement of autophagosomes mediates efficient encounters with lysosomes. Cell. Struct. Funct. 33, 109-122 (2008).

23. Ikenaka, K. et al. dnc-1/dynactin 1 knockdown disrupts transport of autophagosomes and induces motor neuron degeneration. PloS One 8, e54511 (2013).

24. Cheng, X. T., Zhou, B., Lin, M. Y., Cai, Q. \& Sheng, Z. H. Axonal autophagosomes recruit dynein for retrograde transport through fusion with late endosomes. J. Cell. Biol. 209, 377-386 (2015).

25. Kochl, R., Hu, X. W., Chan, E. Y. \& Tooze, S. A. Microtubules facilitate autophagosome formation and fusion of autophagosomes with endosomes. Traffic 7, 129-145 (2006).

26. Maday, S., Wallace, K. E. \& Holzbaur, E. L. Autophagosomes initiate distally and mature during transport toward the cell soma in primary neurons. J. Cell. Biol. 196, 407-417 (2012).

27. Stenmark, H. Rab GTPases as coordinators of vesicle traffic. Nat. Rev. Mol. Cell Biol. 10, 513-525 (2009).

28. Jordens, I. et al. The Rab7 effector protein RILP controls lysosomal transport by inducing the recruitment of dynein-dynactin motors. Curr. Biol. 11, 1680-1685 (2001). 
29. Wijdeven, R. H., Jongsma, M. L., Neefjes, J. \& Berlin, I. ER contact sites direct late endosome transport. Bioessays 37, 1298-1302 (2015).

30. Korolchuk, V. I. et al. Lysosomal positioning coordinates cellular nutrient responses. Nat. Cell Biol. 13, 453-460 (2011).

31. Pankiv, S. et al. FYCO1 is a Rab7 effector that binds to LC3 and PI3P to mediate microtubule plus end-directed vesicle transport. J. Cell Biol. 188, 253-269 (2010).

32. Lebrand, C. et al. Late endosome motility depends on lipids via the small GTPase Rab7. EMBO J. 21, 1289-1300 (2002).

33. Rocha, N. et al. Cholesterol sensor ORP1L contacts the ER protein VAP to control Rab7-RILP-p150 glued and late endosome positioning. J. Cell Biol. 185, 1209-1225 (2009)

34. Sugii, S. et al. Roles of endogenously synthesized sterols in the endocytic pathway. J. Biol. Chem. 281, 23191-23206 (2006).

35. Lu, F. et al. Identification of NPC1 as the target of U18666A, an inhibitor of lysosomal cholesterol export and Ebola infection. eLife 4, e12177 (2015).

36. Hansen, T. E. \& Johansen, T. Following autophagy step by step. BMC. Biol. 9, 39 (2011).

37. Vihervaara, T. et al. Sterol binding by OSBP-related protein 1L regulates late endosome motility and function. Cell. Mol. Life Sci. 68, 537-551 (2011).

38. Weber-Boyvat, M., Kentala, H., Peranen, J. \& Olkkonen, V. M. Liganddependent localization and function of ORP-VAP complexes at membrane contact sites. Cell. Mol. Life Sci. 72, 1967-1987 (2015).

39. Helle, S. C. et al. Organization and function of membrane contact sites. Biochim. Biophys. Acta 1833, 2526-2541 2013.

40. Johansson, M. et al. Activation of endosomal dynein motors by stepwise assembly of Rab7-RILP-p150Glued, ORP1L, and the receptor betall spectrin. J. Cell Biol. 176, 459-471 (2007).

41. Bains, M., Zaegel, V., Mize-Berge, J. \& Heidenreich, K. A. IGF-I stimulates Rab7-RILP interaction during neuronal autophagy. Neurosci. Lett. 488, 112-117 (2011)

42. Burkhardt, J. K., Echeverri, C. J., Nilsson, T. \& Vallee, R. B. Overexpression of the dynamitin (p50) subunit of the dynactin complex disrupts dyneindependent maintenance of membrane organelle distribution. J. Cell Biol. 139, 469-484 (1997)

43. Klionsky, D. J., Eskelinen, E. L. \& Deretic, V. Autophagosomes, phagosomes, autolysosomes, phagolysosomes, autophagolysosomes... wait, I'm confused. Autophagy 10, 549-551 (2014).

44. Sarkar, S. et al. Impaired autophagy in the lipid-storage disorder Niemann-Pick type C1 disease. Cell Rep. 5, 1302-1315 (2013).

45. Fraldi, A. et al. Lysosomal fusion and SNARE function are impaired by cholesterol accumulation in lysosomal storage disorders. EMBO J. 29, 3607-3620 (2010).

46. Balderhaar, H. J. \& Ungermann, C. CORVET and HOPS tethering complexes coordinators of endosome and lysosome fusion. J. Cell Sci. 126, 1307-1316 (2013).

47. Progida, C. et al. RILP is required for the proper morphology and function of late endosomes. J. Cell Sci. 120, 3729-3737 (2007).

48. Plemel, R. L. et al. Subunit organization and Rab interactions of Vps-C protein complexes that control endolysosomal membrane traffic. Mol. Biol. Cell 22, 1353-1363 (2011).

49. Ostrowicz, C. W. et al. Defined subunit arrangement and rab interactions are required for functionality of the HOPS tethering complex. Traffic 11, 1334-1346 (2010).

50. Fu, M. M., Nirschl, J. J. \& Holzbaur, E. L. LC3 binding to the scaffolding protein JIP1 regulates processive dynein-driven transport of autophagosomes. Dev. Cell 29, 577-590 (2014).

51. Cai, Q. et al. Snapin-regulated late endosomal transport is critical for efficient autophagy-lysosomal function in neurons. Neuron 68, 73-86 (2010).

52. Caviston, J. P., Zajac, A. L., Tokito, M. \& Holzbaur, E. L. Huntingtin coordinates the dynein-mediated dynamic positioning of endosomes and lysosomes. Mol. Biol. Cell 22, 478-492 (2011)

53. Wong, Y. C. \& Holzbaur, E. L. The regulation of autophagosome dynamics by huntingtin and HAP1 is disrupted by expression of mutant huntingtin, leading to defective cargo degradation. J. Neurosci. 34, 1293-1305 (2014).

54. Ge, L., Melville, D., Zhang, M. \& Schekman, R. The ER-Golgi intermediate compartment is a key membrane source for the LC3 lipidation step of autophagosome biogenesis. eLife 2, e00947 (2013).

55. Rai, A. et al. Dynein clusters into lipid microdomains on phagosomes to drive rapid transport toward lysosomes. Cell 164, 722-734 (2016).

56. de Saint-Jean, M. et al. Osh4p exchanges sterols for phosphatidylinositol 4-phosphate between lipid bilayers. J. Cell Biol. 195, 965-978 (2011).
57. Mesmin, B. et al. A four-step cycle driven by $\mathrm{PI}(4) \mathrm{P}$ hydrolysis directs sterol/PI(4)P exchange by the ER-Golgi tether OSBP. Cell 155, 830-843 (2013).

58. Tong, J., Yang, H., Yang, H., Eom, S. H. \& Im, Y. J. Structure of Osh3 reveals a conserved mode of phosphoinositide binding in oxysterol-binding proteins. Structure. 21, 1203-1213 (2013).

59. van der Kant, R., Zondervan, I., Janssen, L. \& Neefjes, J. Cholesterol-binding molecules MLN64 and ORP1L mark distinct late endosomes with transporters ABCA3 and NPC1. J. Lipid Res. 54, 2153-2165 (2013).

60. Alpy, F. et al. STARD3 or STARD3NL and VAP form a novel molecular tether between late endosomes and the ER. J. Cell Sci. 126, 5500-5512 (2013).

61. Sancak, Y. et al. Ragulator-Rag complex targets mTORC1 to the lysosomal surface and is necessary for its activation by amino acids. Cell 141, 290-303 (2010).

62. Yu, L. et al. Termination of autophagy and reformation of lysosomes regulated by mTOR. Nature 465, 942-946 (2010).

63. Guo, B. et al. O-GlcNAc-modification of SNAP-29 regulates autophagosome maturation. Nat. Cell Biol. 16, 1215-1226 (2014).

64. Miettinen, T. P. \& Bjorklund, M. Mevalonate pathway regulates cell size homeostasis and proteostasis through autophagy. Cell Rep. 13, 2610-2620 (2015).

65. Tamura, A. \& Yui, N. $\beta$-Cyclodextrin-threaded biocleavable polyrotaxanes ameliorate impaired autophagic flux in Niemann-Pick type C disease. J. Biol. Chem. 290, 9442-9454 (2015).

66. Van Wesenbeeck, L. et al. Involvement of PLEKHM1 in osteoclastic vesicular transport and osteopetrosis in incisors absent rats and humans. J. Clin. Invest. 117, 919-930 (2007)

67. van der Wel, N. et al. M. tuberculosis and M. leprae translocate from the phagolysosome to the cytosol in myeloid cells. Cell 129, 1287-1298 (2007).

68. Calafat, J. et al. Human monocytes and neutrophils store transforming growth factor-alpha in a subpopulation of cytoplasmic granules. Blood 90, 1255-1266 (1997).

69. Ovesny, M., Krizek, P., Borkovec, J., Svindrych, Z. \& Hagen, G. M. ThunderSTORM: a comprehensive ImageJ plug-in for PALM and STORM data analysis and super-resolution imaging. Bioinformatics. 30, 2389-2390 (2014).

\section{Acknowledgements}

We thank the NKI Digital Microscopy Facility for support, members of the Neefjes group for critical discussions, Nico Ong for developing electron micrographs and Wim van $\mathrm{Hu}$ for providing the PLEKHM1 construct. This work was supported by the Institute of Chemical Immunology (ICI), an NWO Gravitation project funded by the Ministry of Education, Culture and Science of the government of the Netherlands and a European Research Council (ERC) advanced grant awarded to J.N.

\section{Author contributions}

R.H.W. and J.N. designed experiments, R.H.W. performed experiments, I.B. performed the live-cell imaging experiments, H.J. performed the Immuno-EM experiments, L.N. and K.J. performed the super-resolution microscopy experiments and L.J. cloned constructs. R.H.W., I.B. and J.N. wrote the manuscript with input from all authors.

\section{Additional information}

Supplementary Information accompanies this paper at http://www.nature.com/ naturecommunications

Competing financial interests: The authors declare no competing financial interests.

Reprints and permission information is available online at http://npg.nature.com/ reprintsandpermissions/

How to cite this article: Wijdeven, R. H. et al. Cholesterol and ORP1L-mediated ER contact sites control autophagosome transport and fusion with the endocytic pathway. Nat. Commun. 7:11808 doi: 10.1038/ncomms11808 (2016).

This work is licensed under a Creative Commons Attribution 4.0 International License. The images or other third party material in this article are included in the article's Creative Commons license, unless indicated otherwise in the credit line; if the material is not included under the Creative Commons license, users will need to obtain permission from the license holder to reproduce the material. To view a copy of this license, visit http://creativecommons.org/licenses/by/4.0/ 